Research Paper

\title{
Chidamide increases the sensitivity of Non-small Cell Lung Cancer to Crizotinib by decreasing c-MET mRNA methylation
}

\author{
Nan Ding, Abin You, Wei Tian, Liankun Gu, Dajun Deng ${ }^{\circledR}$ \\ Key Laboratory of Carcinogenesis and Translational Research (Ministry of Education/Beijing), Division of Etiology, Peking University Cancer Hospital \&
} Institute, Fu-Cheng-Lu \#52, Haidian District, Beijing, 100142, China.

$\square$ Corresponding author: Professor Dajun Deng, Key Laboratory of Carcinogenesis and Translational Research (Ministry of Education/Beijing), Division of Etiology, Peking University Cancer Hospital \& Institute, Fu-Cheng-Lu \#52, Haidian District, Beijing, 100142, China. Phone: +8610-8816-96752; Fax: +8610-88122437; E-mail: dengdajun@bjmu.edu.cn.

(C) The author(s). This is an open access article distributed under the terms of the Creative Commons Attribution License (https://creativecommons.org/licenses/by/4.0/). See http://ivyspring.com/terms for full terms and conditions.

Received: 2020.03.27; Accepted: 2020.07.10; Published: 2020.07.19

\begin{abstract}
Introduction: Crizotinib is a kinase inhibitor targeting c-MET/ALK/ROS1 used as the first-line chemical for the treatment of non-small cell lung cancer (NSCLC) with ALK mutations. Although c-MET is frequently overexpressed in $35-72 \%$ of NSCLC, most NSCLCs are primarily resistant to crizotinib treatment.

Method: A set of NSCLC cell lines were used to test the effect of chidamide on the primary crizotinib resistance in vitro and in vivo. Relationships between the synergistic effect of chidamide and c-MET expression and RNA methylation were systemically studied with a battery of molecular biological assays.

Results: We found for the first time that chidamide could sensitize the effect of crizotinib in a set of ALK mutation-free NSCLC cell lines, especially those with high levels of c-MET expression. Notably, chidamide could not increase the sensitivity of NSCLC cells to crizotinib cultured in serum-free medium without hepatocyte growth factor (HGF; a c-MET ligand). In contrast, the addition of HGF into the serum-/HGF-free medium could restore the synergistic effect of chidamide. Moreover, the synergistic effect of chidamide could also be abolished either by treatment with c-MET antibody or siRNA-knockdown of c-MET expression. While cells with low or no c-MET expression were primarily resistant to chidamide-crizotinib cotreatment, enforced c-MET overexpression could increase the sensitivity of these cells to chidamide-crizotinib cotreatment. Furthermore, chidamide could decrease c-MET expression by inhibiting mRNA N6-methyladenosine (m6A) modification through the downregulation of METTL3 and WTAP expression. Chidamide-crizotinib cotreatment significantly suppressed the activity of c-MET downstream molecules.

Conclusion: Chidamide downregulated c-MET expression by decreasing its mRNA m6A methylation, subsequently increasing the crizotinib sensitivity of NSCLC cells in a c-MET-/HGF-dependent manner.
\end{abstract}

Key words: chidamide; crizotinib; c-MET; non-small cell lung cancer; drug resistance

\section{Introduction}

Non-small cell lung cancer (NSCLC) is the leading cause of cancer death worldwide [1]. Although the recent development of a series of molecular targeted drugs has improved the quality of life and survival of NSCLC patients, a large proportion of NSCLC patients could not benefit from these targeted drugs due to resistance. Therefore, drugs that can reverse primary and secondary drug resistance are urgently expected.

Crizotinib is an ATP-competitive inhibitor targeting ALK/ROS1/c-MET kinases that has been approved by the FDA as a first-line chemical for the treatment of NSCLC and other cancers with ALK rearrangement, ROS1 rearrangement, or aberrant activation of c-MET [2-5]. However, the incidence of these alterations in NSCLCs is relatively low (ALK, 3-7\%; ROS1, 1-2\%; c-MET, 0.8-4\%) [4-6], and the majority of NSCLC patients are primarily resistant to treatment with crizotinib [7]. 
Table 1. The statuses of related gene mutations* and IC50 values $(\mu M)^{* *}$ of chidamide, crizotinib for 13 NSCLC cell lines with or without chidamide co-treatment

\begin{tabular}{|c|c|c|c|c|c|c|c|c|c|c|}
\hline Cell line & $\begin{array}{l}A L K \\
\text { mutation }\end{array}$ & $\begin{array}{l}\text { ROS1 } \\
\text { mutation }\end{array}$ & $\begin{array}{l}\text { EGFR } \\
\text { mutation }\end{array}$ & $\begin{array}{l}\text { K-RAS } \\
\text { mutation }\end{array}$ & $\begin{array}{l}c-M E T \\
\text { amplif. }\end{array}$ & chidamide IC50 (A) & crizotinib IC50 (B) & crizotinib IC50*** (C) & IC50 Fold change ( $\mathrm{C}$ to $\mathrm{B})$ & $\begin{array}{l}\text { t-test, } p \text {-value } \\
\text { (B vs. C) }\end{array}$ \\
\hline EBC-1 & free & free & free & free & positive & $1.24 \pm 0.37$ & $0.004 \pm 0.00$ & $0.003 \pm 0.00$ & $0.75 \pm 0.10$ & 0.011 \\
\hline HCC 827 & free & free & positive & free & free & $0.90 \pm 0.08$ & $0.93 \pm 0.21$ & $0.49 \pm 0.09$ & $0.54 \pm 0.07$ & 0.007 \\
\hline H661 & free & free & free & free & free & $5.51 \pm 0.77$ & $1.10 \pm 0.45$ & $0.62 \pm 0.22$ & $0.58 \pm 0.06$ & 0.006 \\
\hline Calu-3 & free & free & free & free & free & $0.79 \pm 0.10$ & $4.22 \pm 2.22$ & $1.98 \pm 1.42$ & $0.44 \pm 0.08$ & 0.007 \\
\hline H1299 & free & free & free & free & free & $4.09 \pm 0.16$ & $1.34 \pm 0.13$ & $1.10 \pm 0.17$ & $0.82 \pm 0.05$ & 0.026 \\
\hline $\mathrm{H} 358$ & free & free & free & positive & free & $1.73 \pm 0.32$ & $1.46 \pm 0.61$ & $1.13 \pm 0.41$ & $0.79 \pm 0.07$ & 0.041 \\
\hline H596 & free & free & free & free & free & $12.6 \pm 0.05$ & $4.98 \pm 1.56$ & $4.03 \pm 0.92$ & $0.81 \pm 0.07$ & 0.044 \\
\hline $\mathrm{H} 460$ & free & free & free & positive & free & $5.99 \pm 0.65$ & $1.84 \pm 0.26$ & $1.59 \pm 0.23$ & $0.86 \pm 0.00$ & 0.001 \\
\hline A549 & free & free & free & positive & free & $8.25 \pm 1.41$ & $3.21 \pm 1.51$ & $3.50 \pm 1.37$ & $1.11 \pm 0.09$ & 0.166 \\
\hline H1975 & free & free & positive & free & free & $1.85 \pm 0.06$ & $1.86 \pm 0.66$ & $1.94 \pm 0.88$ & $1.03 \pm 0.11$ & 0.706 \\
\hline H1650 & free & free & positive & free & free & $92.5 \pm 15.7$ & $2.17 \pm 0.57$ & $2.25 \pm 0.61$ & $1.03 \pm 0.03$ & 0.166 \\
\hline H292 & free & free & free & free & free & $5.46 \pm 0.30$ & $1.24 \pm 0.11$ & $1.66 \pm 0.28$ & $1.33 \pm 0.13$ & 0.048 \\
\hline H1395 & free & free & free & free & free & $1.05 \pm 0.01$ & $4.91 \pm 2.29$ & $4.85 \pm 2.26$ & $0.99 \pm 0.01$ & 0.140 \\
\hline
\end{tabular}

*According to CCLE databases [27]; **Mean \pm SD; ***Cotreated with chidamide at $1 / 4$ IC50.

Histone deacetylase inhibitors (HDACIs) can increase the acetylation levels of histone and nonhistone proteins, consequently modulating cancer cell proliferation, apoptosis, differentiation, migration, the host immune response and angiogenesis [8, 9]. Several HDACIs, including vorinostat, panobinostat, and chidamide, have been approved for the treatment of cutaneous T-cell lymphoma, multiple myeloma, and peripheral T-cell lymphoma [10-12]. However, therapeutic effect of HDACIs as single agent is very limited for patients with solid tumors.

Fortunately, increasing evidence suggests that HDACIs combined with other agents show a synergetic increase in antitumor activities [13]. For example, HDACIs could sensitize cancer cells to DNA-damaging drugs by the epigenetic activation of Schlafen-11 (SLFN11) [14]. The HDACI FA17 could restore the response of breast cancer cells to methotrexate by inhibiting the activity of nucleophosmin and drug efflux pumps through the PI3K/AKT pathway [15]. The HDACI LAQ824 could downregulate $B C R-A B L$ and sensitize imatinib (an $A B L$ kinase inhibitor) in chronic myelogenous leukemiablast crisis cells [16]. Several studies have also suggested that HDACIs could enhance the effect of EGFR inhibitors in NSCLC by repressing the expression or phosphorylation of EGFR, HER2, c-MET, AXL, and IGF1R [17-19]. Combinations of HDAC6/8 inhibitors with crizotinib could efficiently inhibit diffuse large B-cell lymphoma and neuroblastoma cells [20, 21]. These phenomena suggest that HDACIs could sensitize cancers to different types of drugs and have good application prospects.

Chidamide is a novel HDACI targeting HDAC1/ $2 / 3 / 10$ [22]. In this study, we reported for the first time that chidamide could increase the sensitivity of NSCLC cells to crizotinib in a $c-M E T$ expression- dependent manner in vitro and in vivo. Our further studies unveiled that chidamide could decrease $c-M E T$ expression, probably via the downregulation of the RNA methyltransferase WTAP and METTL3 expression and the subsequent loss of $c-M E T$ m6A mRNA.

\section{Materials and Methods}

\section{Cell lines and culture}

In this study, thirteen NSCLC cell lines without ALK mutations and HGF expression were used (Table 1 and Figure S1). H1299 cells were kindly provided by professor Chengchao Shou, and A549 cells (with KRAS mutations) were kindly provided by professor Zhiqian Zhang. EBC-1 cell line with c-MET gene amplification (kindly provided by Dr. Yue Yang) was used as a crizotinib-sensitive control [23]. These two cell lines were tested and authenticated by Beijing JianLian Genes Technology Co., Ltd. before they were used in this study. STR patterns were analyzed using the Goldeneye 20A STR Identifier PCR Amplification Kit. Gene Mapper v3.2 software (ABI) was used to match the STR pattern with those in the online databases of the American Type Culture Collection (ATCC). The other ten cell lines (HCC827, Calu-3, H661, H596, H358, H460, H1650, H1975, H1395, and H292) were purchased from the National Laboratory Cell Resource Sharing Platform (Beijing, China) at the beginning of this study with STR authentications.

Calu-3 cells were cultured in MEM with 1\% nonessential amino acids, EBC- 1 cells were cultured in MEM with $1 \%$ nonessential amino acids and $1 \%$ sodium pyruvate $(100 \mathrm{mM}), \mathrm{H} 596$ cells were cultured in DMEM, and the other ten cell lines were cultured in RPMI 1640 medium. These cell lines, cultured at $37^{\circ} \mathrm{C}$ in a humidified incubator with $5 \% \mathrm{CO}_{2}$, were all supplemented with $10 \%$ fetal bovine serum (FBS) and 
$100 \mathrm{U} / \mathrm{mL}$ penicillin/streptomycin (Invitrogen, CA, USA).

\section{Reagents}

Crizotinib (CAS No. 877399-52-5) and ricolinostat (CAS No. 1316214-52-4) were purchased from Selleck Chemicals (Houston, TX, USA). Chidamide (CAS No. 743420-02-2) was kindly provided by Shenzhen Chipscreen Biosciences Ltd. (Shenzhen, China). Human hepatocyte growth factor (HGF; Entrez Gene 3082) was purchased from Sino Biological, Inc. (10463-HNAS, Beijing, China).

\section{Cell viability assay}

Cells were seeded into 96-well plates $\left(3-10 \times 10^{3}\right.$ cells per well according to different cell growth rates, 5 wells per group) and incubated overnight. Twenty-four hours postseeding, the cells were treated with crizotinib at various concentrations alone or combined with chidamide at an inhibitory concentration of $1 / 4$ IC50 (final concentration, $\leq 1.0$ $\mu \mathrm{M})$. After treatment for $72 \mathrm{hr}$, the proliferation status of these cells was dynamically determined with a long-term observation platform (IncuCyte, Essen, MI, USA). The cell confluence was analyzed using IncuCyte ZOOM software. Cell viability percent $(\%)=$ (cell confluence percent of experimental group/cell confluence percent of control group) $\times 100 \%$. The half maximal inhibitory concentration (IC50) values were calculated by GraphPad Prism 6 (GraphPad Software, Inc., San Diego, USA). The combination index (CI) values were calculated by CompuSyn version 2.0 software (Biosoft, Cambridge, UK). CI $<1.0$ indicates a synergistic effect or overcoming of resistance; $\mathrm{CI}=$ 1.0 indicates an additive effect; and CI $>1.0$ indicates an antagonistic effect of two drugs [23]. All experiments were conducted three times independently. The IC50 fold change was the ratio of crizotinib-IC50 with and without chidamide and was used to represent the synergistic effect between chidamide and crizotinib.

\section{Cell apoptosis assay}

Approximately $2 \times 10^{6}$ cells were plated in $10-\mathrm{cm}$ dishes. After the cells attached, they were exposed to DMSO, crizotinib, chidamide or the combination of the two drugs for $48 \mathrm{hr}$. Then, the cell suspension was collected and incubated with Annexin V-FITC and PI in the dark for $15 \mathrm{~min}$ according to the protocol of the Annexin V-Fluorescein Isothiocyanate (FITC) Apoptosis Kit (Dojindo, Kumamoto, Japan). These samples were analyzed by flow cytometry (BD Biosciences, NJ, USA) within $1 \mathrm{hr}$.

\section{Cell colony assay}

Cells were seeded in 6-well plates at a density of
$10^{3}$ cells per well. After the cells attached, they were treated with DMSO, crizotinib, chidamide or the combination of the two drugs. The medium containing drugs was replaced twice a week for 10-20 days. Finally, the cells were fixed with methyl alcohol and stained with $0.1 \%$ crystal violet.

\section{RNA extraction and quantitative RT-PCR (qRT-PCR) assay}

Total RNA was extracted using an Ultrapure RNA kit (Beijing Com Win Biotech Co., China) and reverse-transcribed into cDNA using the First-Strand cDNA Synthesis Kit (Transgen Co., Beijing, China). Then, the cDNA samples were amplified by qRT-PCR using SYBR Green PCR master mix reagents (Roche, Mannheim, Germany) in triplicate. The relative expression levels of the detected genes were normalized to that of the GAPDH reference RNA calculated by the classical $\Delta \Delta \mathrm{Ct}$ method. The sequences $\left(5^{\prime}-3^{\prime}\right)$ of the primers used are as follows: c-MET (Entrez Gene 4233; forward, ccaccctttgttcagtgt gg; and reverse, agtcaaggtgcagctctcat), ALK (Entrez Gene 238; forward, gcctgtggctgtcagtatttg; and reverse, tcccatagcagcactccaaag), ROS1 (Entrez Gene 6098; forward, aggctgccaacatgtctgat; and reverse, cggccagat ggtacaggaag), WTAP (Entrez Gene 9589; forward, taa agcaacaacagcaggag; and reverse, aatagtccgacgccatca), METTL3 (Entrez Gene 56339; forward, agtgacagccca gtgcctac; and reverse, acagtccetgctacctccc), GAPDH (Entrez Gene 2597; forward, gagatggtgatgggatttc; and reverse, gaaggtgaaggtcggagt), and $18 \mathrm{~S}$ rRNA (forward, gagatggtgatgggatttc; and reverse, gaaggtga aggtcggagt).

\section{Western blotting}

The protein lysates from treated cells were run on an $8 \%$ SDS-PAGE gel and transferred onto a PVDF membrane. Then, the membrane was blocked with 5\% fat-free milk overnight at $4{ }^{\circ} \mathrm{C}$. The next day, the membrane was incubated with the primary antibodies (MET(D-4)/sc-514148, p-MET(F-5)/sc-377548, STAT3 (F-2)/sc-8019, p-STAT3(B-7)/sc-8059, Santa Cruz, USA; AKT(pan) (C67E7)/\#4691, p-AKT/\#406, ERK(1/2) (137F5)/\#4695， p-ERK(Thr202/Tyr204) (D13.14.4E)/\#4370, WTAP/\#5650, METTL3 (D2I6O)/ \#96391， METTL14(D8K8W)/\#51104， EGFR/\#2232, pEGFR(Y1068)/\#2234, Cell Signaling Technology, USA; FTO/ab126605, Abcam, UK; and GAPDH/ 60004-1, Protein Tech, China) at room temperature for at least $1 \mathrm{hr}$. Then, the membrane was washed with PBST ( $1 \times$ PBS with $0.1 \%$ Tween 20$)$ three times at an interval of $10 \mathrm{~min}$. After washing, the membrane was incubated with the appropriate goat anti-rabbit (SE131, Solarbio, China) or goat anti-mouse (SE131, Solarbio, China) secondary antibodies at room 
temperature for $1 \mathrm{hr}$. After washing 6 times, the signals were visualized using the Immobilon Western Chemiluminescent HRP Substrate Kit (WBKLS0500, Millipore, Billerica, USA).

\section{Plasmids and siRNA transfection}

The pLenti-MetGFP vector was kindly provided by David Rimm (Addgene plasmid \# 37560; http:/ / n2t.net/addgene: 37560; RRID: Addgene_37560) [24]. The empty vector was constructed by deleting the targeted gene from the pLenti-MetGFP vector. HEK293FT cells were seeded in $6 \mathrm{~cm}$ plates before transfection, and when the cell confluence reached $40 \%$, they were transfected with the pLenti-MetGFP vector or the control vector using the lentiviral packaging kit (BG20401S, Beijing Syngentech Co., China) according to the manufacturer's manual. Then, the cell supernatant, filtered by a $0.45 \mu \mathrm{m}$ needle filter, was collected and used to infect A549 and H1650 cells $48 \mathrm{hr}$ posttransfection. Afterward, the infected cells were cultured in medium containing $1 \mu \mathrm{g} / \mathrm{mL}$ puromycin (Sigma, St. Louis, USA) for at least two weeks to obtain stably transfected cells.

The plasmids p3xflag-cmv-14-XN-hWTAP and pCMV-myc-XN-hMETTL3 were kindly provided by Professor Yun-Gui Yang [25]. Cells were transfected with the plasmid using X-tremeGENE HP DNA Transfection Reagent (Roche, Mannheim, Germany) according to the manufacturer's manual when the cells reached a confluence of $50-70 \%$. And shRNAs for WTAP and METTL3 were synthesized by GenePharma Co. (Shanghai, China). The successful knockdown and overexpression of WTAP and METTL3 was confirmed by Western blotting.

The siRNA oligo sequences $\left(5^{\prime}-3^{\prime}\right)$ against c-MET mRNAs (\#1: sense, gguguugucucaauaucaatt; antisense, uugauauugagacaacacctt; \#2: sense, gcaacagcug aaucugcaatt; antisense, uugcagauucagcuguugctt), WTAP (\#1: sense, cacagaucuuaacucuaautt; antisense, auuagaguuaagaucugugtt; \#2: sense, gacccagcgaucaac uugutt; antisense, acaaguugaucgcuggguctt), METTL3 (\#1: sense, gcacauccuacucuuguaatt; antisense, uuacaa gaguaggaugugctt; \#2: sense, gacugcucuuuccuuaauatt; antisense, uauuaaggaaagagcaguctt) were synthesized by GenePharma Co. (Shanghai, China). HCC827 and H661 cells were transfected with the siRNAs using X-tremeGENETM siRNA Transfection Reagent (Roche, Mannheim, Germany) according to the manufacturer's manual when the cells reached a confluence of $60-80 \%$. The successful knockdown of c-MET expression was confirmed by Western blotting and qRT-PCR. Scramble siRNAs (sense, 5'-uucuccga acgugucacgutt- $3^{\prime}$; antisense, $5^{\prime}$-acgugacacguucggaga att- $3^{\prime}$ ) were used as the negative control. Cells were further used in other experiments $48 \mathrm{hr}$ post- transfection.

\section{RNA N6-methyladenosine (m6A) dot blotting}

Total RNA was extracted from cells in a 6-well microplate (one well per group) using the RNA isolation kit mentioned above. Then, equal amounts of RNA samples were denatured by formaldehyde and spotted on a nylon membrane. After the UV crosslinking of 2500J, the membrane was blocked with a $1 \%$ blocking solution (Cat. 11096176001, Roche) for $1 \mathrm{hr}$. Then, the membrane was incubated with anti-m6A antibody (ab151230, Abcam, UK) at room temperature for at least $2 \mathrm{hr}$. After washing with 1\% blocking solution three times at an interval of $10 \mathrm{~min}$, the membrane was incubated with anti-rabbit secondary antibody (same as above) at room temperature for 1 hr. The input control was dyed by methylthioninium chloride immediately after UV cross-linking.

\section{m6A methylated RNA immunoprecipitation assay (MeRIP)}

Cells in four $10 \mathrm{~cm}$ dishes were collected after $\mathrm{CHI}$ treatment for $4 \mathrm{hr}$. The MeRIP assay was carried out using the RNA-Binding Protein Immunoprecipitation Kit (Cat\# 17-701, EZ-Magna, Millipore, USA) according to the manufacturer's instructions. Total m6A-containing RNAs were immunoprecipitated and extracted using m6A antibody (the same as above for m6A dot blotting). cDNA was synthesized from the RIP-RNAs using random primers, and gene-specific quantitative PCR was then performed using the c-MET or 18S rRNA control primers in triplicate. The relative c-MET mRNA copy number was calculated using that of the $18 S$ rRNA as an internal reference.

\section{Animal experiments}

HCC827 $\left(3 \times 10^{6}\right)$ cells suspended in $100 \mu \mathrm{L}$ of PBS were injected into the left inguen of female Balb/c nude mice (body weight 18-20 g; age 6 weeks; Beijing Huafukang Bioscience Co., Inc.). When the tumor volumes reached $50-100 \mathrm{~mm}^{3}$ on the 10th posttransplantation day, the mice were randomized into four groups (10 mice per group) and were intragastrically administered vehicle (normal saline), crizotinib (25 mg/ $\mathrm{kg}$ body weight), chidamide (5 $\mathrm{mg} / \mathrm{kg}$ ), or the combination of the two drugs daily for 21 days. The tumor volumes and body weights of the mice were measured every 3 days. Tumor volumes were calculated using the following formula: tumor volume $\left(\mathrm{mm}^{3}\right)=\left[(\right.$ length $\left.) \times(\text { width })^{2}\right] / 2$. These mice were sacrificed by dislocation of cervical vertebra on the 21st treatment day. The CI value in this animal experiment was calculated as follows: $\mathrm{CI}=[(\mathrm{cA}+$ $\mathrm{cB})-\mathrm{cA} \times \mathrm{cB}] / \mathrm{cAB}$. $\mathrm{cA}$ represents the inhibitory rate of chidamide alone, $c B$ represents the inhibitory 
rate of crizotinib alone, and $\mathrm{CAB}$ represents the inhibitory rate of the combination treatment of chidamide and crizotinib. Synergy is defined as a CI value lower than 1.0 [26].

All experiments were performed in accordance with institutional standard guidelines of Peking University Cancer Hospital and adhered to the U.S. Public Health Service Policy on the Humane Care and Use of Laboratory Animals.

\section{Statistical analysis}

SPSS 20.0 software (Armonk, NY, USA) was used to perform the statistical analysis. The Kolmogorov-Smirnov test was used to estimate the normality of distributions. The Mann-Whitney U test was conducted for non-normally distributed data. Student's t-test was conducted for normally distributed data. All statistical tests were two-sided. $P$ $<0.05$ was considered statistically significant.

\section{Results}

\section{Increasing the sensitivity of NSCLC cells to crizotinib by chidamide}

Twelve NSCLC cell lines (HCC827, H661, Calu-3, H1299, H460, H596, H358, H292, H1650, H1975, H1395, and A549) with the wild type ALK gene were selected according to Pharmacogenomics datasets of the Cancer Cell Line Encyclopedia (CCLE) project [27] and used in this study. First, we examined the cytotoxicity and IC50 value of chidamide as a single agent for these cell lines and found that the effect of chidamide was time- and dose-dependent (range: 0.79 - $92.5 \mu \mathrm{M}$, Table 1; Figure S2A-L). Next, we investigated the antiproliferative activities of crizotinib at various concentrations with and without chidamide at $\leq 1 / 4$ IC50 (final concentration, $\leq 1.0 \mu \mathrm{M}$ ) for each cell line. Compared to crizotinib treatment alone, we found that chidamide-crizotinib cotreatment decreased viability in seven cell lines (HCC827, H661, Calu-3, H1299, H460, H596, and H358; Figure 1A-G, top charts). In these cells, the chidamide-crizotinib $\mathrm{CI}$ values were $<1.0$, and the crizotinib IC50 values for the chidamide (at 1/4 IC50)-treated cell lines were significantly lower than those treated with crizotinib alone (HCC827, $p=0.007$; H661, $p=0.006$; Calu-3, $p=0.007$; H1299, $p=0.026$; $\mathrm{H} 358, p=0.041$; H596, $p=0.044$; and H460, $p<0.001$ ) (Figure 1A-G, bottom chart). Especially for the chidamide-cotreated HCC827, H661, and Calu-3 cell lines, the CI values were $<0.7$, and the crizotinib IC50 values decreased approximate $50 \%$ (Table 1). For the H292, H1650, H1975, H1395, and A549 cell lines, the synergistic effect between crizotinib and chidamide was not seen, and the CI values were $\geq 1$ (Figure
1H-L). An antagonistic effect was detected in H292 cells $(p=0.048)$. These data revealed that chidamide could synergistically enhance the cytotoxic effects of crizotinib in 7 of the 12 NSCLC cell lines with primary crizotinib resistance.

To further validate the chidamide-crizotinib synergistic effect, cell apoptosis and colony formation assays were performed using the HCC827, Calu-3, and H661 cell lines. Compared with single drug treatment, chidamide-crizotinib cotreatment significantly increased the percentage of apoptotic cells $(67.7 \%$ for HCC $827 ; 58.7 \%$ for H661; and $34.6 \%$ for Calu-3; $p<0.050$; Figure 2A). Chidamide-crizotinib cotreatment also significantly inhibited colony formation in all of these cell lines $(6.7 \%$ for HCC 827 ; $3.9 \%$ for H661; and $8.6 \%$ for Calu-3 cells; $p<0.050$; Figure 2B).

Overall, these data suggested that chidamidecrizotinib cotreatment apparently promoted the cell apoptosis and inhibited the cell proliferation of NSCLC cells compared with single drug treatment in vitro.

\section{Enhancement of crizotinib sensitivity by chidamide in a c-MET expression-dependent manner}

To explore the underlying mechanism of enhancing the sensitivity of NSCLC cells to crizotinib by chidamide, the baseline mRNA levels of the crizotinib target genes $A L K$, ROS1 and c-MET were determined. We found that in cell lines in which chidamide could enhance the sensitivity of crizotinib, the level of c-MET expression was much higher than that in cell lines in which chidamide could not increase the sensitivity of crizotinib (Figure 3A). The level of c-MET mRNA was inversely correlated with the chidamide-based crizotinib-IC50 fold change (the smaller the change value was, the stronger the sensitization effect) $(n=12, r=-0.706, p=0.010$; Figure 3B; Table 1). No significant association was observed between the chidamide-based crizotinib-IC50 fold change and the level of ALK or ROS1 mRNA. The Western blotting results confirmed the qRT-PCR results (Figure $3 \mathrm{C}$ ). Amounts of the c-MET protein and phosphorylated c-MET protein (p-MET) were also inversely correlated with the chidamide-based crizotinib-IC50 fold change $(n=12 ; r=-0.694, p=0.012$ for c-MET; $r=-0.561, p=0.058$ for $p$-MET). No significant relationship between the level of the c-MET protein and crizotinib-IC50 or chidamide-IC50 was found (Figure 3D). These phenomena imply the possibility that the synergistic effect of chidamide on crizotinib sensitivity in NSCLC cells may depend on the level of c-MET expression. 

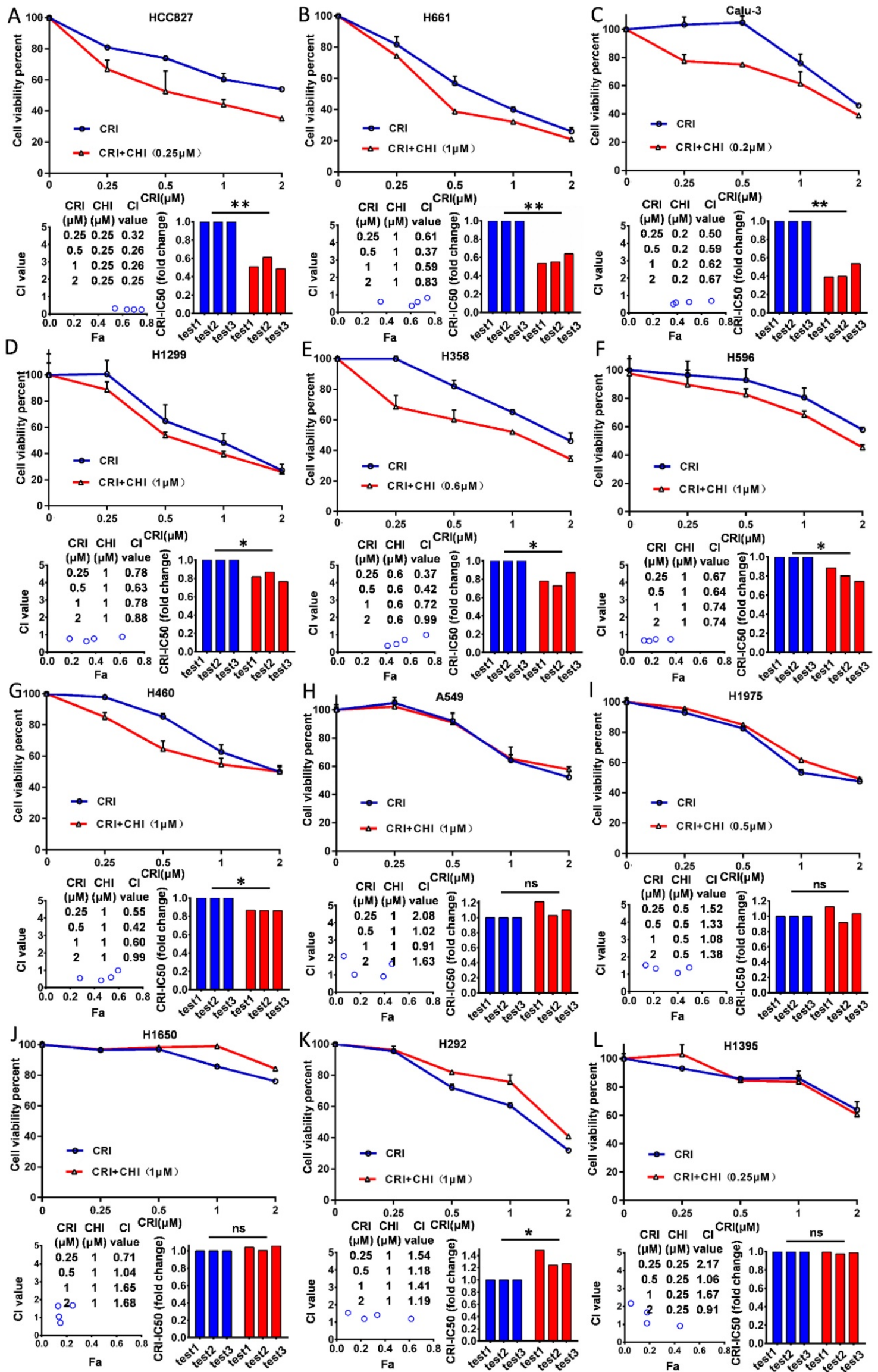

Figure 1. Effect of chidamide on the primary resistance of ALK mutation-free NSCLC cells to crizotinib. NSCLC cells were treated with various concentrations of crizotinib (CRI) alone or combined with chidamide (CHI) at $\leq 1 / 4$ IC50 for $72 \mathrm{hr}$. Cell viability was measured by using the IncuCyte platform (top charts). The crizotinib-IC50 values for 12 
NSCLC cell lines were calculated in the absence or presence of chidamide, and the fold change of crizotinib-IC50 in three independent experiments is presented as a histogram (bottom right charts). The synergetic effect of chidamide-crizotinib cotreatment on cell proliferation inhibition was calculated using the $\mathrm{Cl}$ equation and presented as Fa (fraction affected by the dose) in the Fa-Cl plots (bottom left charts). */**/***, p<0.05/0.0/0.001; ns, not significant; (A) HCC827; (B) H661; (C) Calu-3; (D) HI299; (E) H358; (F) H596; (G) H460; (H) A549; (I) H1975; (J) H1650; (K) H292; (L) H1395.

A
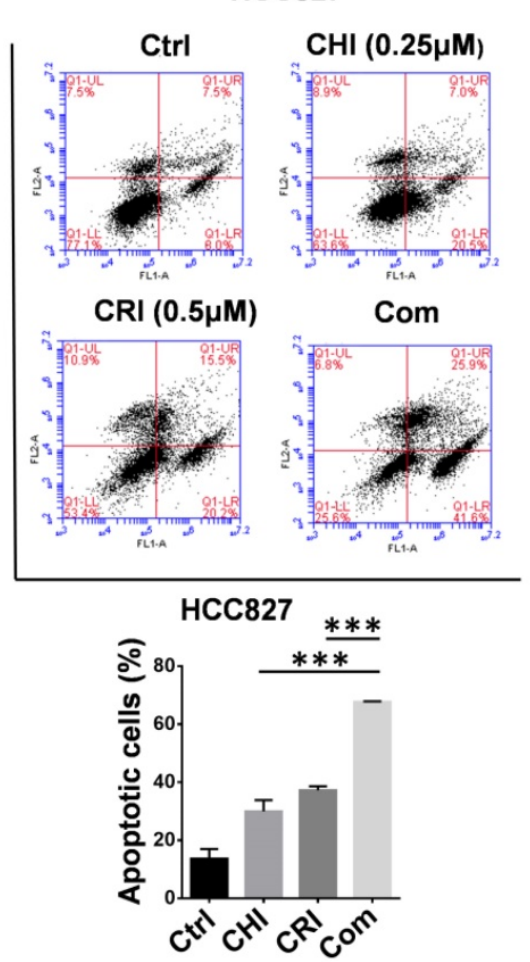

H661
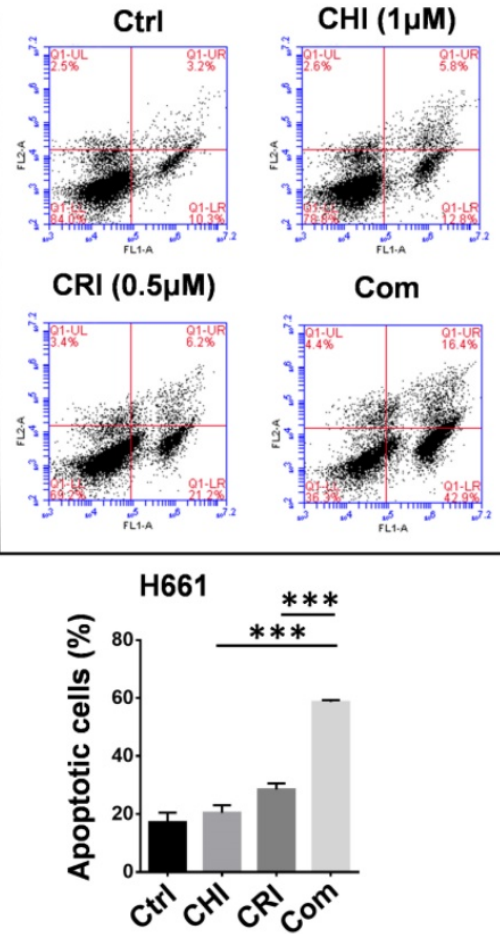

\section{$\mathrm{CHI}(1 \mu \mathrm{M})$}

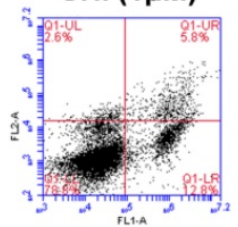

Com
Calu-3

CRI $(1 \mu \mathrm{M})$
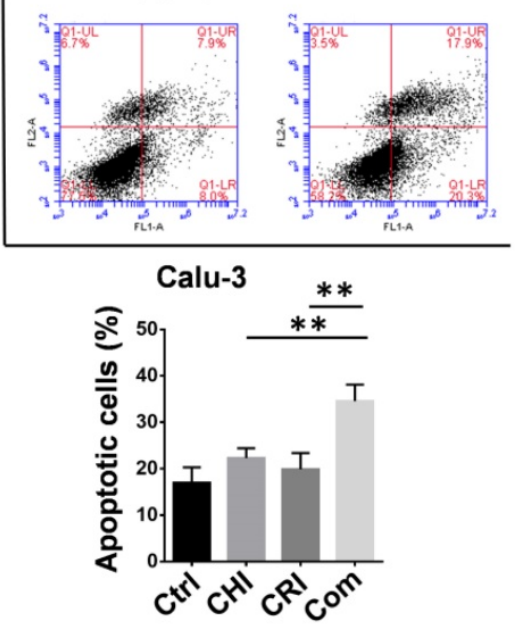

B

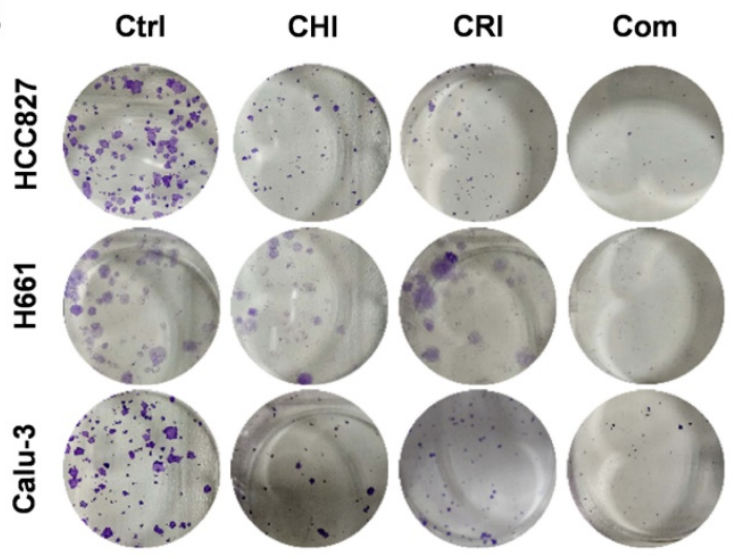

HCC827

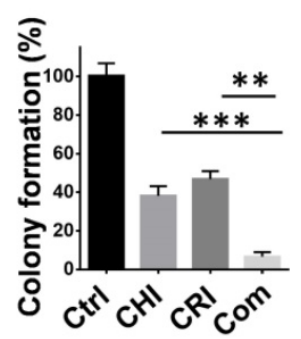

H661

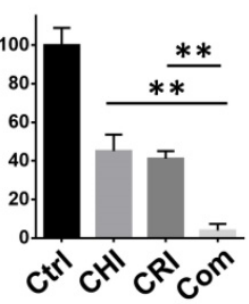

Calu-3

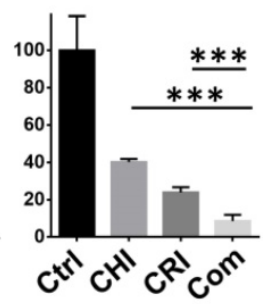

Figure 2. Effect of chidamide on the crizotinib-induced apoptosis and growth arrest of NSCLC cells. (A) Effect of chidamide (CHI), crizotinib (CRI), and chidamide-crizotinib cotreatment on the apoptosis of HCC827, H661, and Calu-3 cells. Cells were treated with chidamide (at $1 / 4$ IC50) and crizotinib $(0.5$ or $1 \mu \mathrm{M}$ ) alone or in combination for 48 hr. DMSO was used as an agent control (Ctrl). Apoptotic cells were labeled with propidium iodide/Annexin V-FITC and analyzed by flow cytometry. (B) Effect of chidamide, crizotinib, and cotreatment on the colony formation of $\mathrm{HCC} 827, \mathrm{H} 661$, and Calu-3 cells. These cells were treated with the indicated concentrations of chidamide or crizotinib for 10-20 days. Colonies were stained with $0.1 \%$ crystal violet and photographed (left images). The results represent the mean of three independent experiments (right charts). $* * * * *, \mathrm{p}<0.01 / 0.001$.

Therefore, we further studied whether c-MET could play a driver role in the synergetic effect of chidamide-crizotinib. In the serum-free medium not containing HGF (a c-MET ligand), no synergetic effect was found in the above chidamide-crizotinibsensitive cell lines HCC827 and H661 with high baseline $c-M E T$ expression. However, the synergetic effect was restored with the addition of HGF (final concentration, $100 \mathrm{ng} / \mathrm{mL}$ ) into the serum-/HGF-free medium (Figure 4A). In addition, the synergetic effect could be reversed in cells either by treatment with c-MET antibody (Figure 4B) or by c-MET knockdown (Figure 4C). Loss of function of c-MET by antibody or HGF-deprivation could also inhibit the proliferation of HCC 827 and H661 cells (Figure S3). 

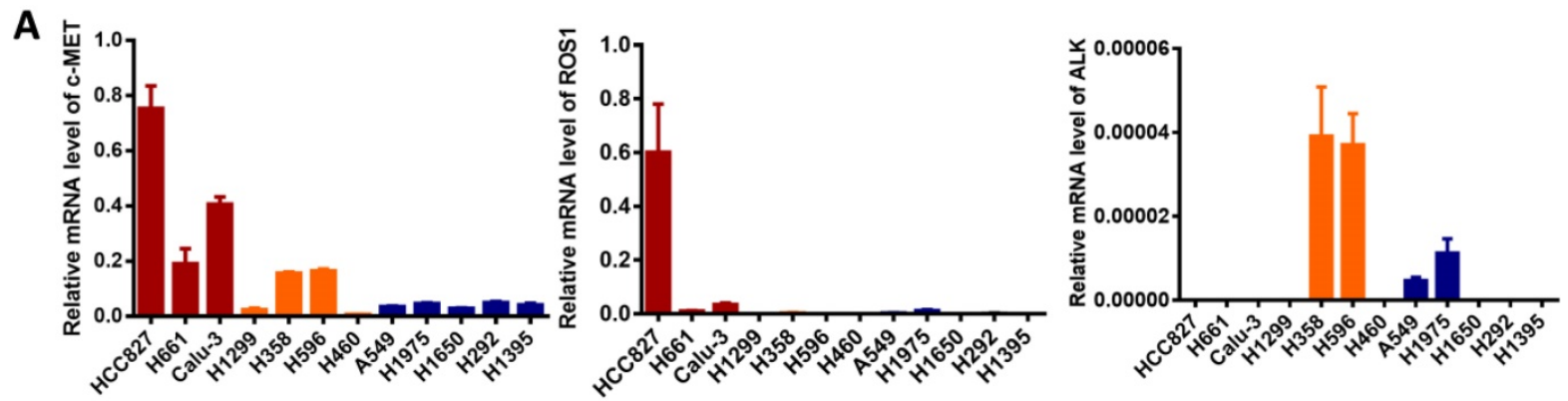

B
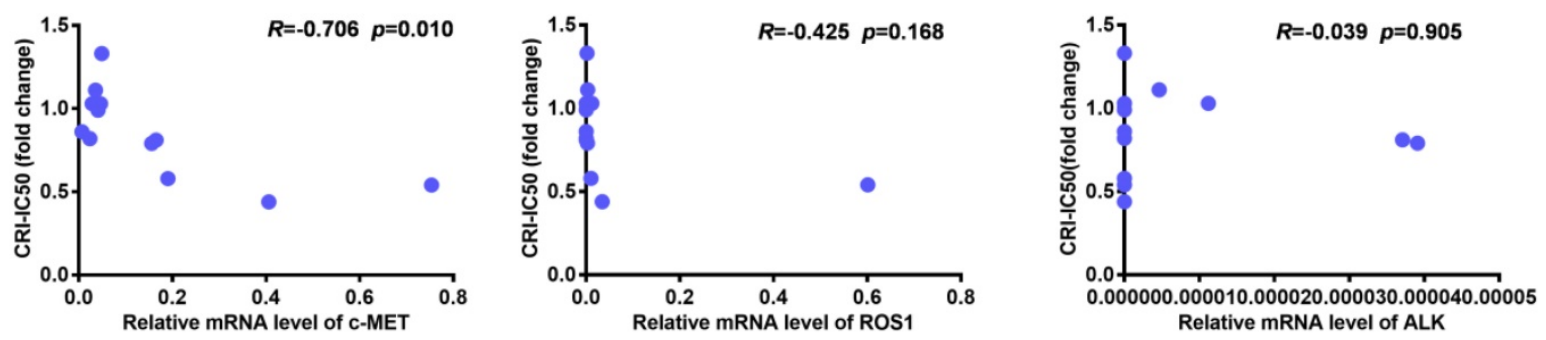

C
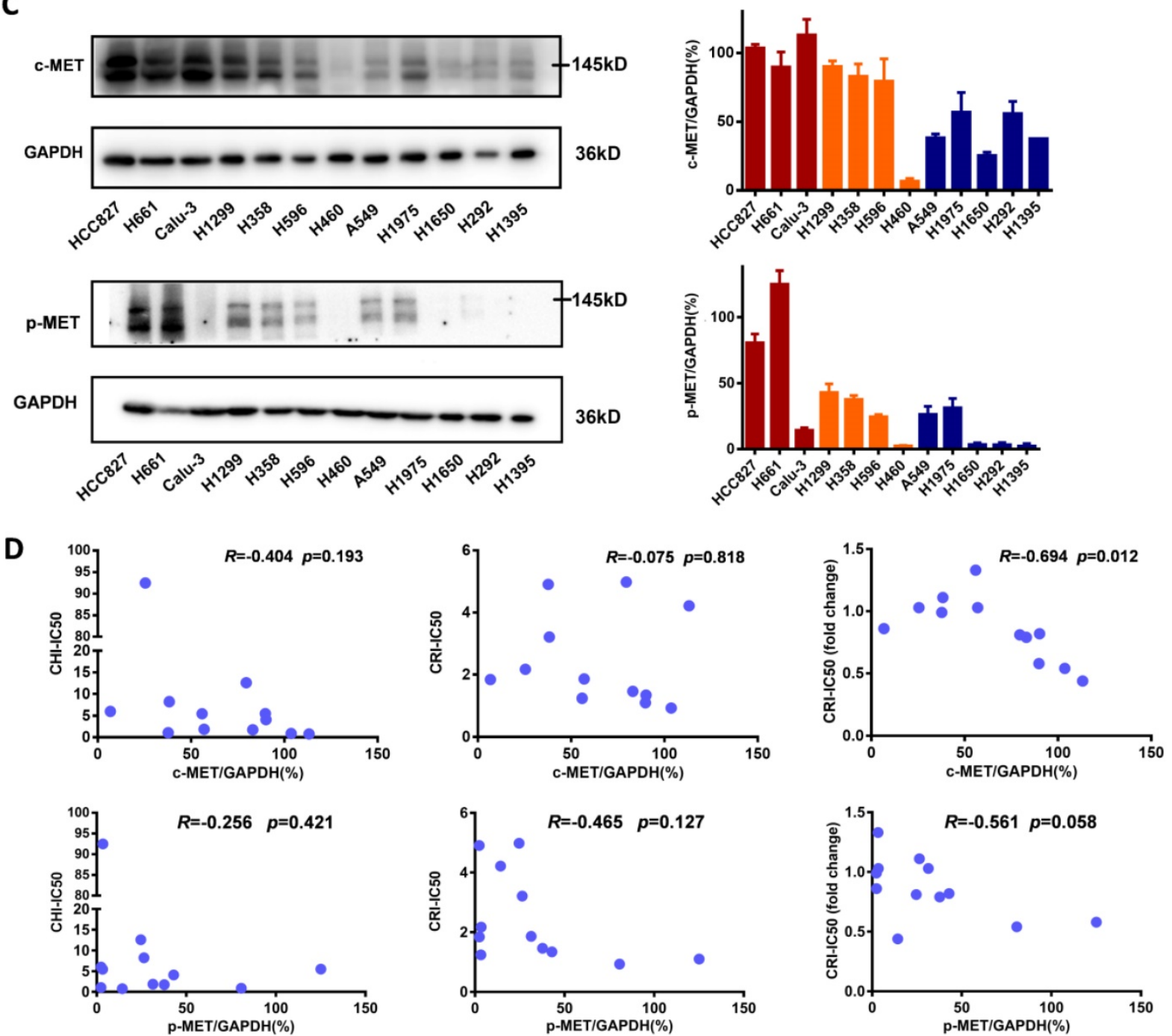

Figure 3. Comparisons of c-MET, ROS1, ALK expression levels in NSCLC cells with different crizotinib sensitivities. (A) The levels of c-MET, ROS1, ALK mRNAs in 12 NSCLC cell lines by qRT-PCR. (B) Relationships between the crizotinib IC50 fold change and baseline mRNA expression levels of c-MET, ROS1, ALK genes in 12 NSCLC cell lines. (C) The baseline levels of c-MET and phosphorylated c-MET (p-MET) proteins in 12 NSCLC cell lines by Western blotting. The density of each band was calculated by Image software, and the ratio of c-MET/GAPDH and p-MET/GAPDH is presented by means with bars representing the SEM. (D) Relationships between the levels of c-MET and p-MET proteins and chidamide IC50, crizotinib IC50 (without chidamide), and crizotinib IC50 fold change (with chidamide) in 12 NSCLC cell lines. Pearson correlation analyses were performed to calculate the correlation coefficient $(R)$ and $p$-value. 


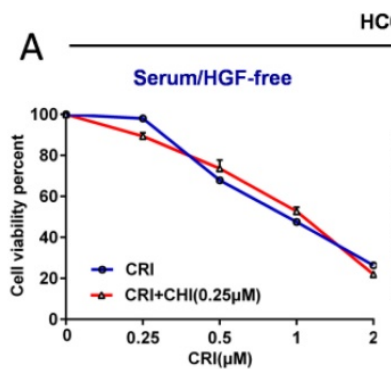

HCC827

B

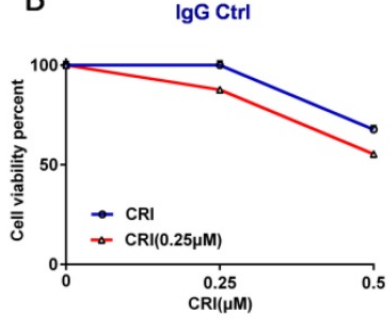

C

Scramble
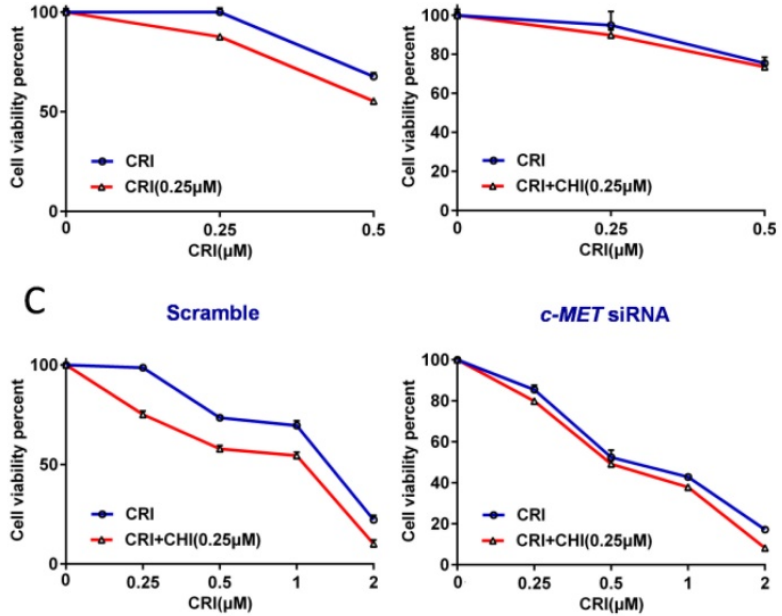

c-MET SIRNA
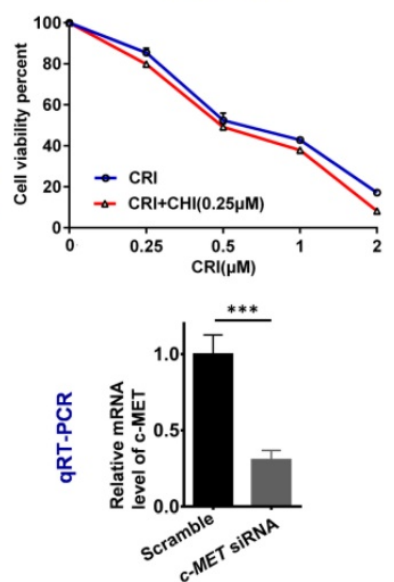

A549
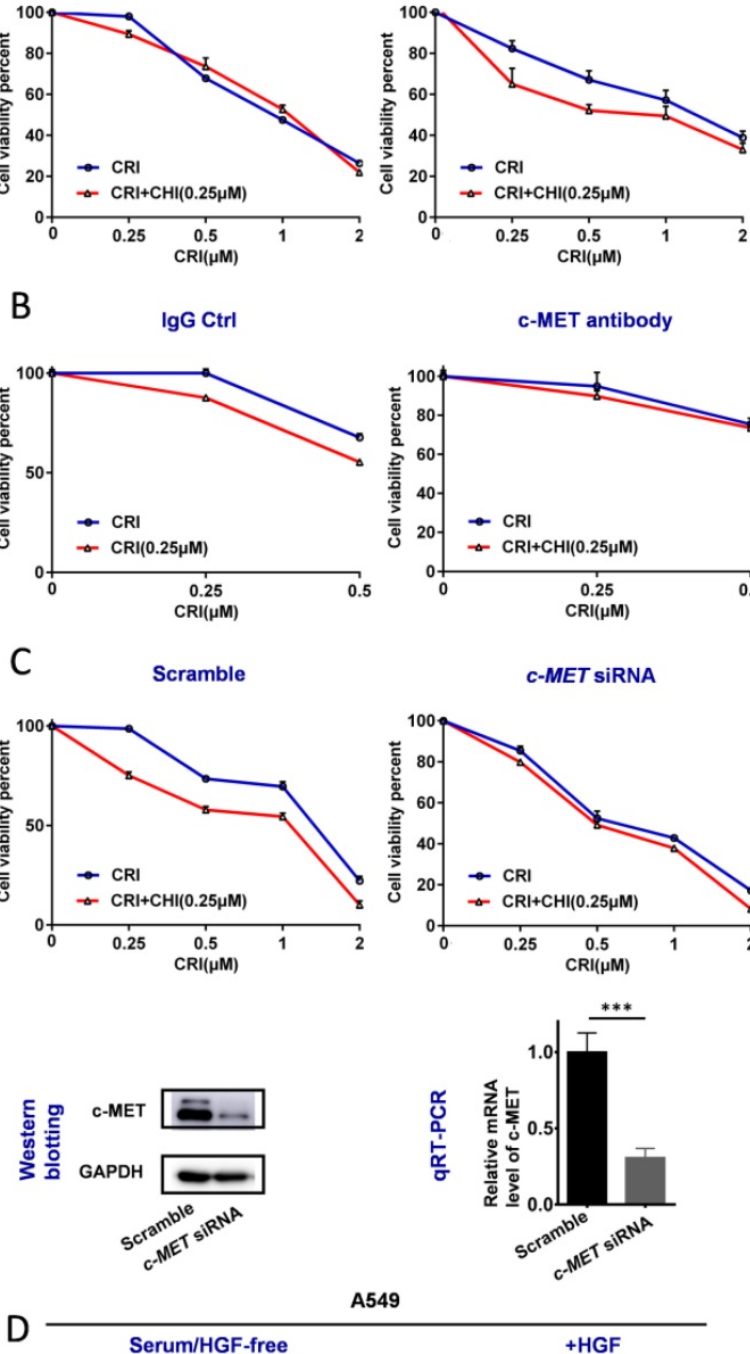

c-MET antibody

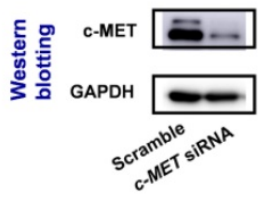

D
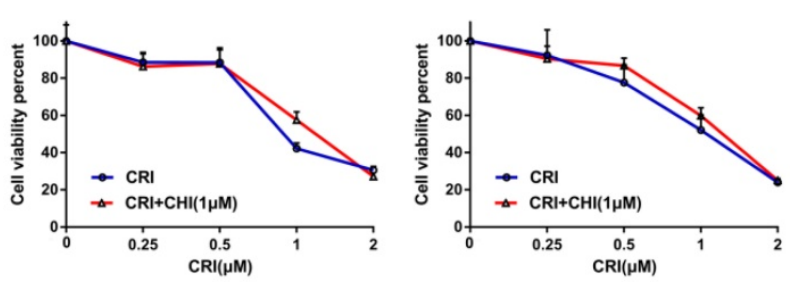

E

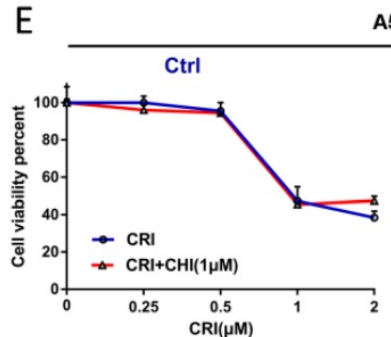

A549

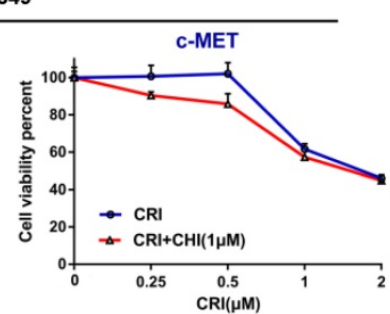

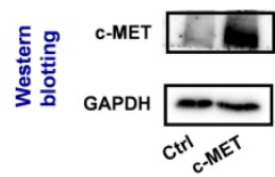

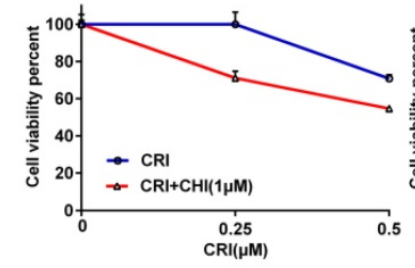

Scramble
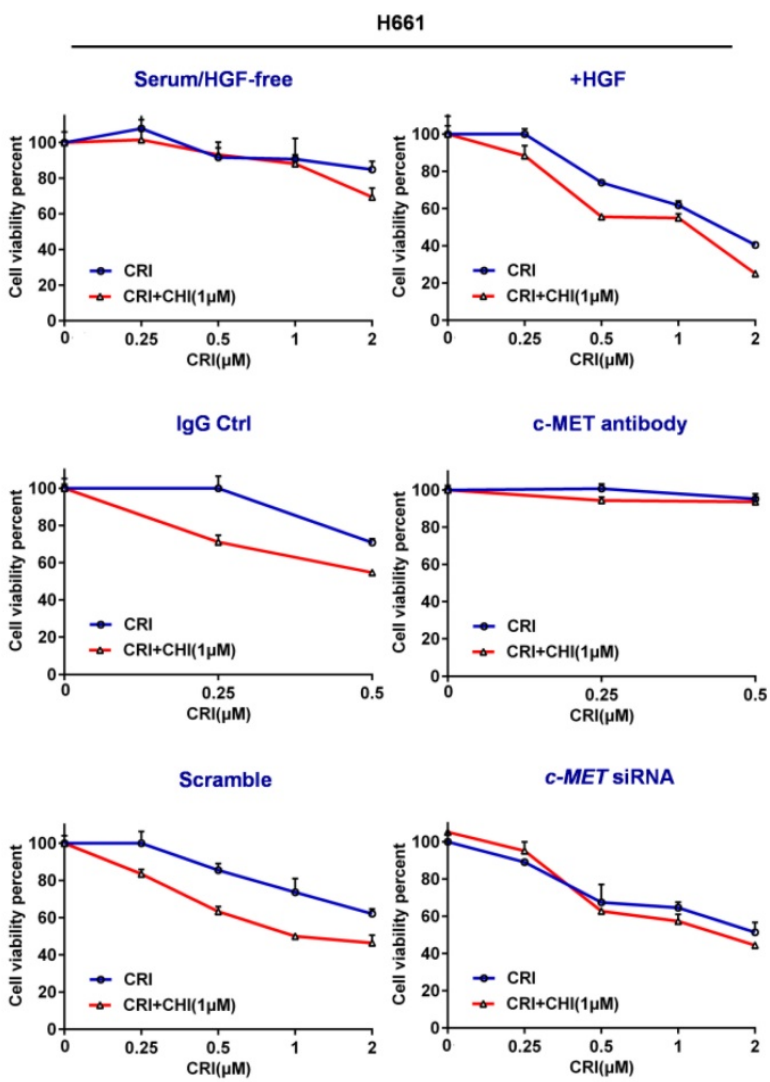

gG Ctr

c-MET antibody

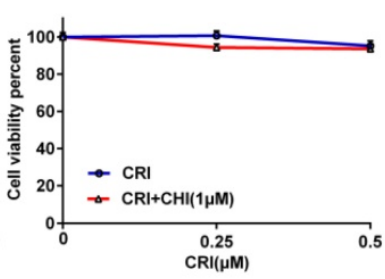

c-MET SIRNA

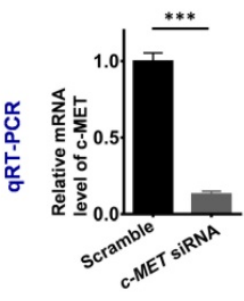

H1650

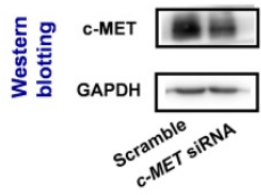

+HGF

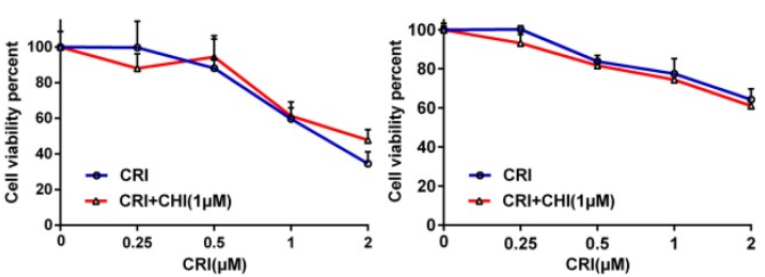

H1650
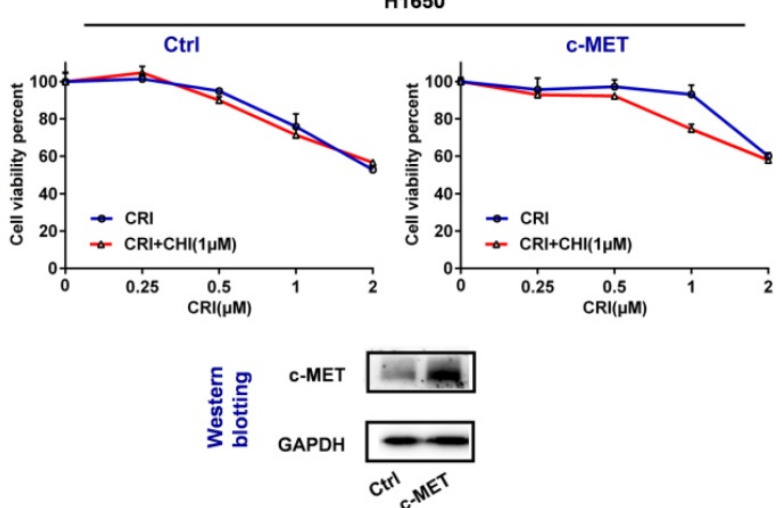

Figure 4. Effect of the loss and gain of c-MET function on increasing the crizotinib sensitivity of NSCLC cells by chidamide. (A) The increasing of crizotinib sensitivity by chidamide in two chidamide-crizotinib-sensitive cell lines, HCC827 and H661, cultured in serum-free medium with no c-MET ligand HGF or with 100 ng/mL HGF supplement. (B) 
Effect of c-MET antibody treatment $(2.5 \mu \mathrm{g} / \mathrm{mL})$ on the increasing of crizotinib sensitivity by chidamide in $\mathrm{HCC} 827$ and $\mathrm{H} 661 \mathrm{cells}$. IgG $(2.5 \mu \mathrm{g} / \mathrm{mL}) \mathrm{was}$ used as a negative antibody control. (C) Effect of siRNA knockdown of c-MET mRNA on the increasing of crizotinib sensitivity by chidamide in HCC 827 and H661 cells. Cells transfected with the scramble siRNA were used as the transfection agent control. (D) Comparison of the increasing of crizotinib sensitivity by chidamide in two chidamide-crizotinib non-sensitive control cell lines (A549 and H1650) cultured in serum-free medium with no HGF or with $100 \mathrm{ng} / \mathrm{mL}$ HGF supplement. (E) Effect of enforced c-MET overexpression on the increasing of crizotinib sensitivity of the A549 and $\mathrm{H} 1650$ cell lines by chidamide.

For the two chidamide-crizotinib-resistant control cell lines A549 and H1650, the synergetic effect of chidamide-crizotinib was not different between the serum-free medium with and without the addition of HGF (Figure 4D). However, enforced c-MET overexpression could induce the synergetic effect of chidamide-crizotinib (Figure 4E). In EBC-1 cells with $c-M E T$ gene amplification, a significant increase of the level of $c-M E T$ mRNA, but not the amount of c-MET protein, was detected relative to those of two control cell lines HCC827 and Calu-3 (Figure S4A-B). Interestingly, the synergetic effect of chidamidecrizotinib was also observed in the representative crizotinib-sensitive EBC- 1 cells and chidamide could downregulate the level of $c-M E T$ expression (Figure S4C-D). Overall, the above data indicate that the synergetic effect of chidamide-crizotinib is dependent on c-MET expression. Low or no c-MET expression leads to the absence of a synergetic effect.

\section{Downregulation of c-MET expression by chidamide via RNA m6A modification}

We further studied the mechanisms of chidamide in increasing crizotinib sensitivity. After treatment with chidamide for $4 \mathrm{hr}$, the amounts of c-MET protein by Western blotting in three chidamide-crizotinib-sensitive cell lines (HCC827, H661 and Calu-3) were significantly decreased while the levels of c-MET mRNA by qRT-PCR were not significantly changed (Figure 5A), suggesting a posttranscriptional mechanism.

To dissect which of the posttranscriptional processes chidamide may affect to influence the amount of c-MET protein, these cell lines were cotreated with several known inhibitors, including actinomycin D (ActD; $5 \mu \mathrm{g} / \mathrm{mL}$ ), cycloheximide (CHX; $50 \mu \mathrm{g} / \mathrm{mL}$ ), and proteasome inhibitor MG132 $(10 \mu \mathrm{M})$, for $4 \mathrm{hr}$ to block RNA transcription, protein synthesis, and degradation, respectively (Figure 5B). Western blotting analyses showed that the downregulation of $c-M E T$ expression by chidamide remained in the cells cotreated with MG132. However, the amount of c-MET protein was not decreased in the cells cotreated with $\mathrm{CHX}$ or ActD, and the level of c-MET mRNA was unchanged by ActD (Figure 5C). These results suggest that both ubiquitin-related protein degradation and RNA transcription may not be involved in downregulation of c-MET expression by chidamide, and chidamide may decrease the amount of c-MET protein from the posttranscription to translation steps.

RNA modifications regulate RNA splicing, translation, and degradation, and $\mathrm{m} 6 \mathrm{~A}$ is the main RNA modification [28]. To study whether chidamide affects c-MET m6A mRNA modification, we compared the levels of global and c-MET m6A mRNA in these cells with and without chidamide treatment. Dot blotting showed that chidamide could markedly decrease the total m6A RNA level of HCC 827 and H661 cells (Figure 6A). The results of the MeRIPbased qRT-PCR assay further demonstrated that a significant decrease in the relative c-MET m6A copy number was induced in the chidamidecrizotinib-sensitive cell lines HCC827 and H661 by chidamide (-1.83 for HCC827; -1.91 for H661; Figure $6 \mathrm{~B})$. Although the total levels of total m6A RNA were also decreased in the two chidamide-crizotinibresistant control cell lines A549 and H1650 by chidamide (Figure 6A), a very limited decrease in the relative c-MET m6A copy number could be induced due to the low baseline level of $c-M E T$ mRNA in these cells (-0.11 for A549; -0.24 for H1650; Figure 6B). This finding demonstrates that chidamide (at 1/4 IC50) treatment may increase crizotinib sensitivity by inhibiting the m6A modification of c-MET mRNA.

To study how chidamide affects RNA m6A modification, the expression levels of several known m6A writer complex components (METTL3, METTL14, and WTAP) and eraser (FTO) were determined. The Western blotting results revealed that the amounts of METTL3 and WTAP proteins were consistently decreased (Figure 6C), and the levels of METTL3 and WTAP mRNAs were not significantly changed in chidamide-treated HCC827 and H661 cells (Figure 6D). In addition, the synergetic effect of chidamide-crizotinib disappeared in both HCC827 and H661 cells with siRNA knockdown of METTL3 or WTAP expression (Figure 6E). In the rescue assay, METTL3 or WTAP overexpression could restore the synergetic effect of chidamide-crizotinib in H661 cells with stable shMETTL3 or shWTAP transfection (Figure S5). These data suggest that the downregulation of METTL3 and WTAP expression may play a crucial role in the increasing of crizotinib sensitivity by chidamide.

In addition, we also checked the effect of chidamide on the expression states of METTL3 and WTAP in HCC827 and H661 cells respectively cotreated with MG132, CHX, and ActD, as described above. We found that no change of the amounts of 
METTL3 and WTAP proteins could be induced by chidamide in these cells cotreated with CHX (Figure 7A). However, the amounts of METTL3 and WTAP proteins was still decreased by chidamide in these cells cotreated with ActD (or MG132) and the mRNA levels of WATP and METTL3 were not changed between the ActD-cotreated cells with and without chidamide treatment (Figure 7B). The results of the MeRIP-based qRT-PCR assay showed a significant decrease of the m6A mRNA levels of WTAP and METTL3 (Figure 7C-D). These results indicate that chidamide could also decrease the stability and translational efficiency of WTAP and METTL3 mRNAs.

\section{Synergistic inhibition of the growth of NSCLC by chidamide-crizotinib cotreatment in mice}

To further evaluate the effect of chidamidecrizotinib cotreatment in vivo, we subcutaneously injected HCC827 cells into the left inguen of female Balb/c mice (10 mice per group). When the tumor volumes reached $50-100 \mathrm{~mm}^{3}$, these mice with established HCC827-derived tumors were treated (i.g.) with vehicle, chidamide and crizotinib alone or their combination for 21 days. We found that while chidamide or crizotinib treatment alone only weakly decreased the tumor weight compared to the vehicle control, chidamide and crizotinib cotreatment led to a markedly decreased growth of tumors on the 21st treatment day (Figure 8A-B). Chidamide-crizotinib cotreatment exhibited ideal synergistic effects $(\mathrm{CI}=$ 0.880). Moreover, chidamide and crizotinib treatment alone or in combination did not lead to a loss of body weight (Figure 8C) or to other apparent illnesses, suggesting high safety. These findings demonstrate that chidamide could increase the sensitivity of NSCLC cells to crizotinib in vivo.
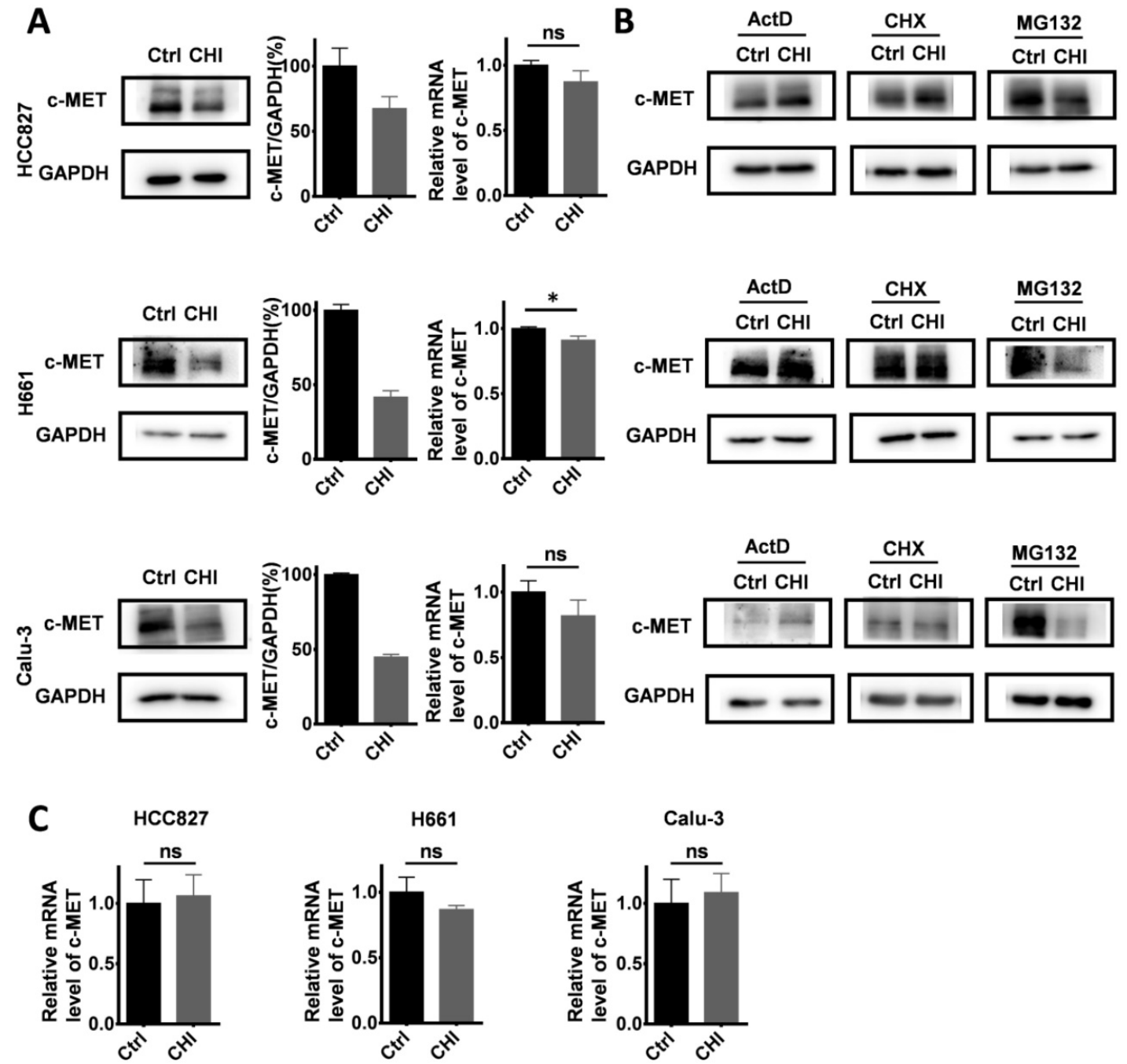

Figure 5. Effect of chidamide on the levels of c-MET expression in NSCLC cells. (A) Effects of chidamide treatment $(0.25 \mu M$ for HCC $827,1 \mu M$ for H661, $0.2 \mu M$ for Calu-3; $4 \mathrm{hr}$ ) on the levels of c-MET expression in HCC827, H661, and Calu-3 cells in Western blotting (left images and middle charts) and qRT-PCR (right charts) analyses. (B) Effects of treatment with transcription, translation, and proteasome inhibitors (ActD, CHX, and MG132) on the chidamide-induced downregulation of c-MET expression in HCC827,

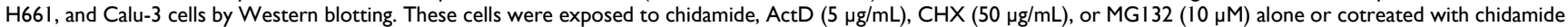
and ActD, CHX, or MG132 for 4 hr. (C) The level of c-MET mRNA in HCC827, H661, and Calu-3 cells cotreated with ActD. *, p<0.05; ns, not statistically significant. 
A
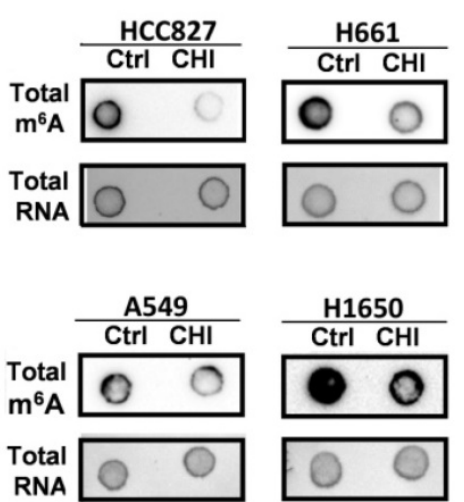

C

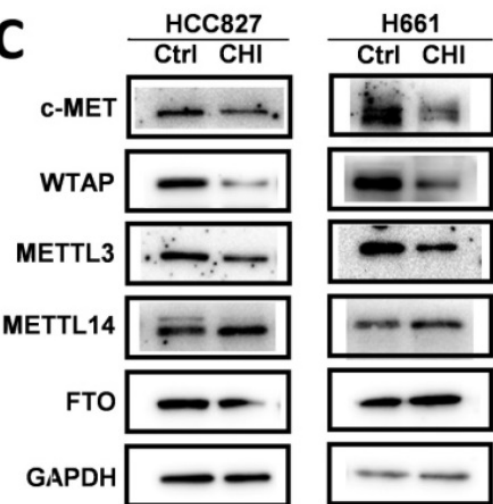

E scramble
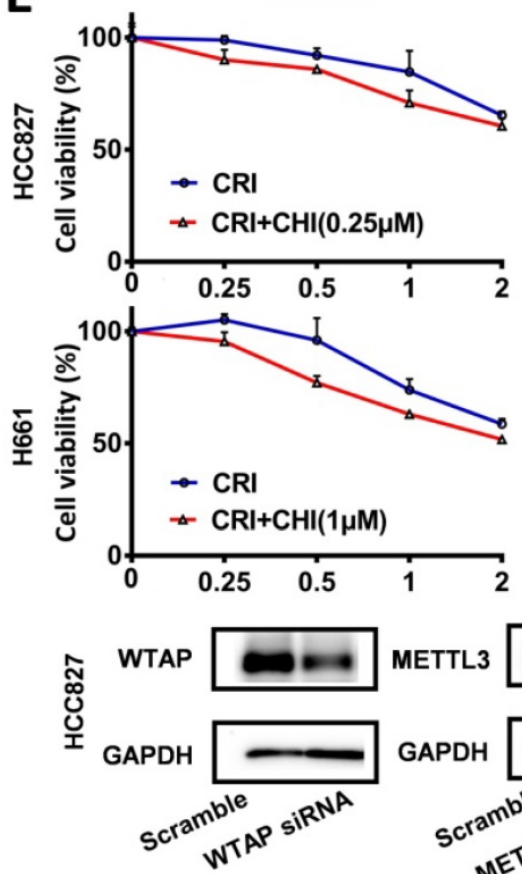
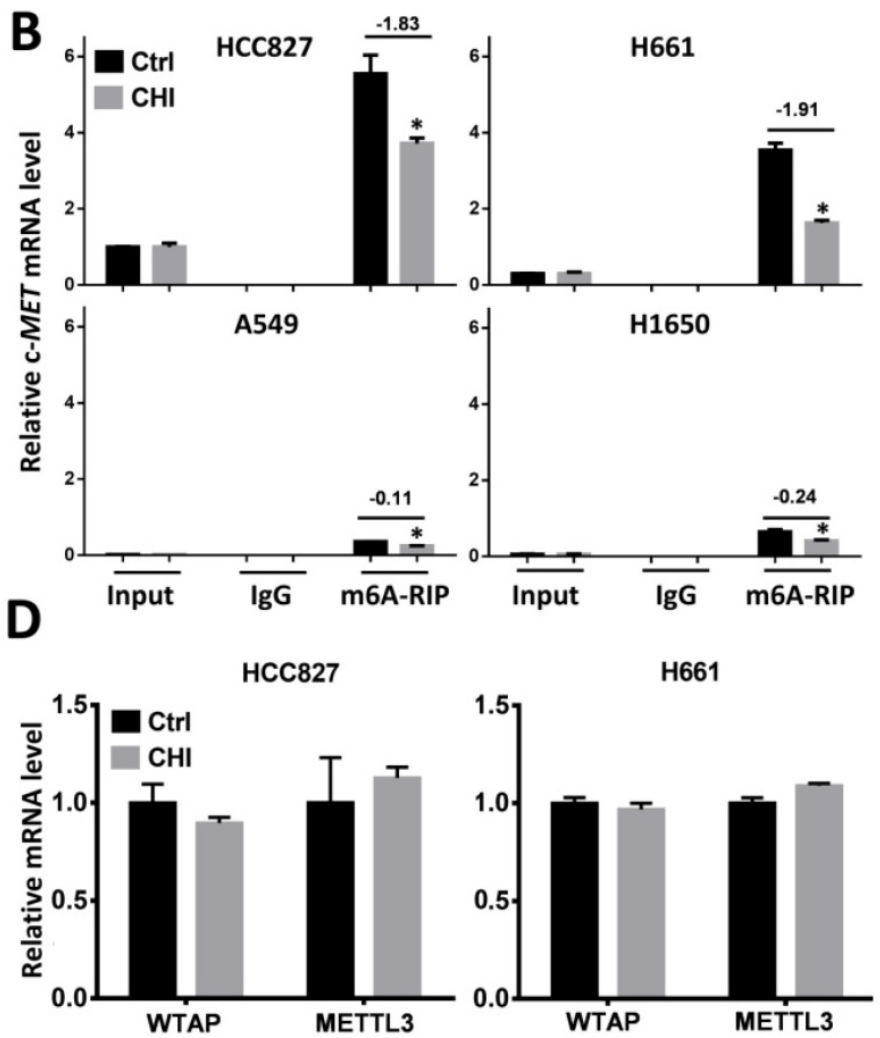

WTAP SiRNA
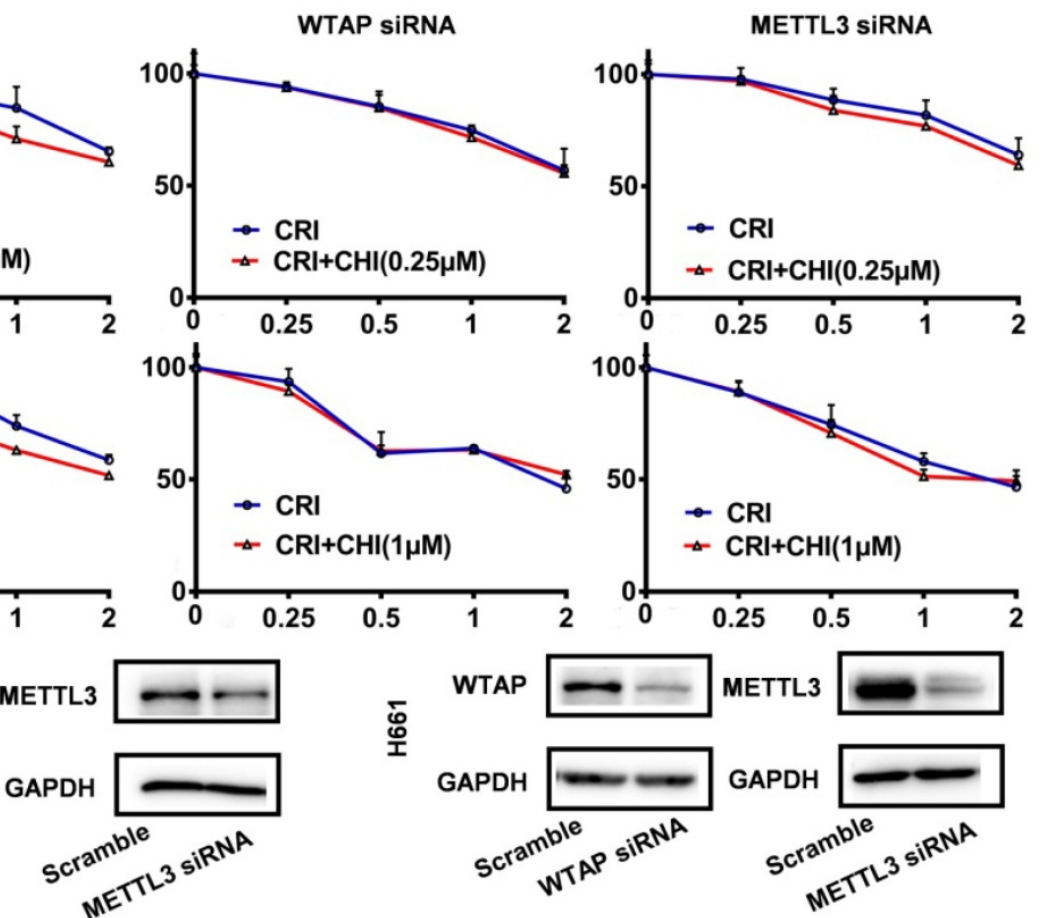

Figure 6. Effect of RNA m6A methylation changes on increasing the crizotinib sensitivity of NSCLC cells by chidamide. (A) The amounts of total m6A RNA of two chidamide-crizotinib-sensitive cell lines $(\mathrm{HCC} 827$ and $\mathrm{H} 661)$ and two chidamide-crizotinib-resistant cell lines (A549 and $\mathrm{H} 1650)$ treated with chidamide $(0.25 \mu \mathrm{M}$ for $\mathrm{HCC} 827$; $1 \mu \mathrm{M}$ for $\mathrm{H661}$, A549, and $\mathrm{H1650;} 4 \mathrm{hr}$ ) in dot blotting analyses. (B) Relative copy number (RCN) of c-MET m6A mRNA in chidamide-crizotinib-sensitive and -resistant cell lines with and without the treatment of chidamide in MeRIP-based qRT-PCR analyses. The average input RCN value of the c-MET mRNA of HCC827 control cells was used to normalize all RCN values of the c-MET mRNA of the 4 cell lines in different groups. The RCN difference of c-MET m6A mRNA between cells with and without chidamide treatment is labeled. (C) Amounts of m6A methylation writer complex components WTAP and METTL3 proteins in HCC827 and H661 cells with and without chidamide treatment in Western blotting. (D) The levels of WTAP and METTL3 mRNAs in chidamide-treated HCC827 and H661 cells. (E) Effect of siRNA knockdown of METTL3 or WTAP expression on the synergetic effect of chidamide-crizotinib in HCC827 and H661 cells. **/***, $\mathrm{p}<0.01 / 0.001$. 


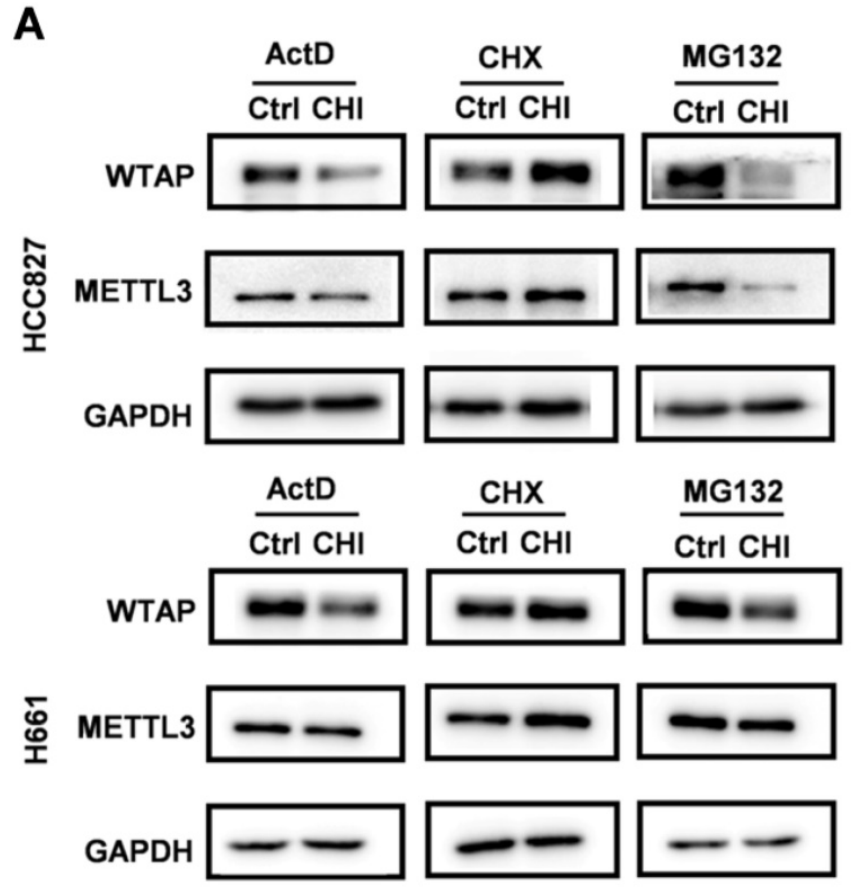

C

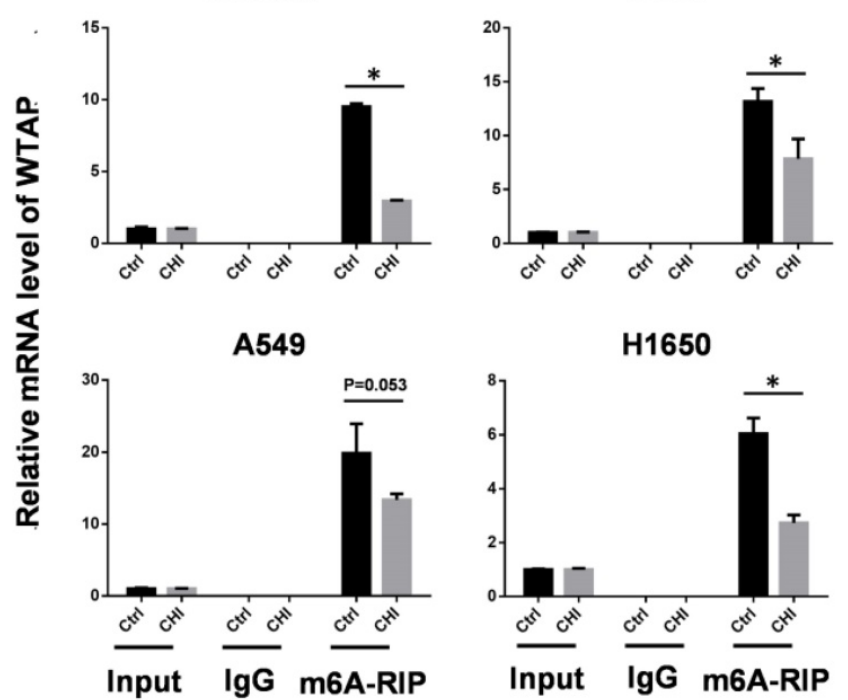

B
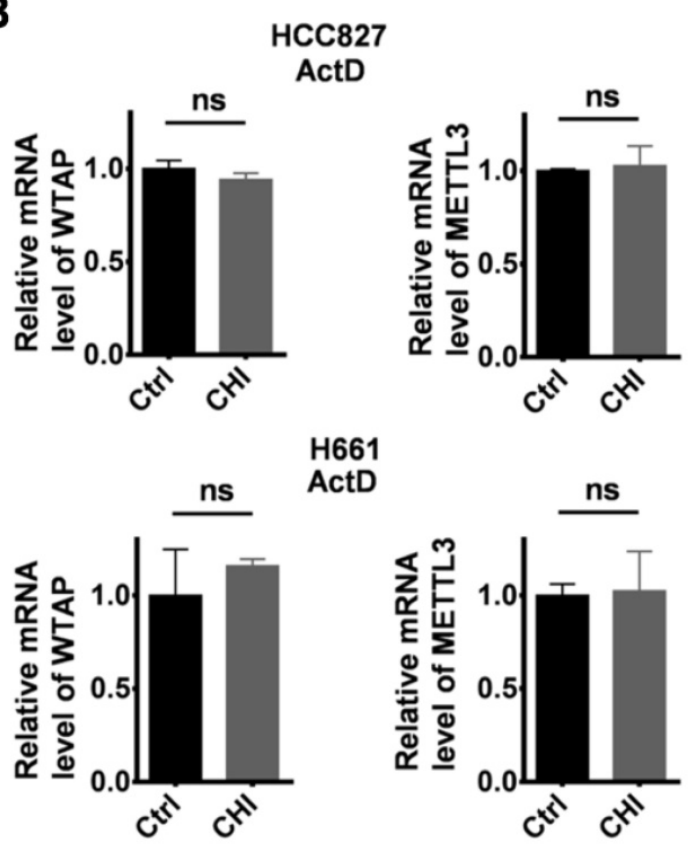

Figure 7. Effect of chidamide on the levels of WTAP and METTL3 expression in NSCLC cells. (A) Effects of treatments with transcription, translation, and proteasome inhibitors (ActD, CHX, and MG132) on the chidamide-induced decrease of the amounts of WTAP and METTL3 proteins in HCC827 and H661 cells in Western blotting. These cells were exposed to ActD $(5 \mu \mathrm{g} / \mathrm{mL}), \mathrm{CHX}(50 \mu \mathrm{g} / \mathrm{mL})$, or MG132 $(10 \mu \mathrm{M})$ alone or cotreated with chidamide for 4 hr. (B) The relative level of WTAP and METTL3 mRNA in HCC827 and $\mathrm{H} 661$ cells cotreated with ActD; (C and D) Relative WTAP and METTL3 m6A mRNA levels in chidamide-crizotinib-sensitive cell lines (HCC827 and H661) and -resistant cell lines (A549 and H1650) with and without the treatment of chidamide in MeRIP-based qRT-PCR analyses. */**, p<0.05/0.01; ns, not statistically significant.

It was reported that crizotinib inhibited tumor growth by decreasing the phosphorylation levels of c-MET, ERK, AKT, and STAT3 [29, 30]. In HCC827 and H661 cell lines with primary crizotinib resistance, crizotinib treatment alone could not affect the phosphorylation levels of ERK and STAT3 proteins in HCC827 cells or the phosphorylation levels of c-MET and ERK proteins in H661 cells. However, the phosphorylation levels of all four of these proteins were consistently decreased in both cell lines with chidamide-crizotinib cotreatment (Figure S6A). The effects of chidamide-crizotinib cotreatment on the phosphorylation of these proteins were also observed in representative mouse HCC827-derived tumors (Figure S6B).

\section{Discussion}

Chidamide is a benzamide HDAC inhibitor approved for the treatment of cutaneous T-cell lymphoma. Studies suggest that chidamide may 
sensitize some DNA damage or target-specific drugs $[10,31,32]$. Crizotinib is a first-line chemical approved for the treatment of NSCLC and other cancers with $A L K$ and ROS1 mutations or c-MET amplification [2-5]. In the present study, we found, for the first time, that chidamide can increase the crizotinib sensitivity of $A L K$ mutation-free NSCLC cells in a c-MET/HGF-dependent manner. Furthermore, the downregulation of RNA m6A writer METTL3 and WTAP expression and consequent loss of c-MET m6A mRNA is the possible mechanism for chidamide to increase the crizotinib sensitivity of NSCLC cells actively expressing c-MET.

The membrane HGF receptor c-MET is a tyrosine kinase that can activate the PI3K-AKT, Ras-MAPK, and STAT3 signaling pathways and promote cancer cell proliferation, migration, and invasion [29, 30, 33]. Recent studies have found that the c-MET-HGF axis also plays an important role in the occurrence and development of NSCLC [34-37]. There are many mechanisms leading to the upregulation of c-MET overexpression, including gene copy number amplification and posttranslational modifications [38]. Although c-MET is one of the targets of crizotinib, only patients with c-MET amplification or exon-14 skipping mutation are currently approved to receive crizotinib treatment. Relative to the low incidence of c-MET amplification, c-MET overexpression occurs in 35-72\% of NSCLCs [38-42]. In our study, we found that the amount of c-MET protein was not associated with the crizotinib IC50 value in 12 NSCLC cell lines. The mining result of the Cancer Cell Line Encyclopedia (CCLE) dataset [27] confirmed our results that no correlation between the level of c-MET mRNA and the crizotinib IC50 value of pan-cancer cell lines (Figure S7A) or lung cancer cell lines could be observed (Figure S7B). While NSCLCs with c-MET amplification or exon14 splicing alterations were responsive to crizotinib treatment, some NSCLCs with high c-MET expression were primarily resistant to crizotinib, which may account

A
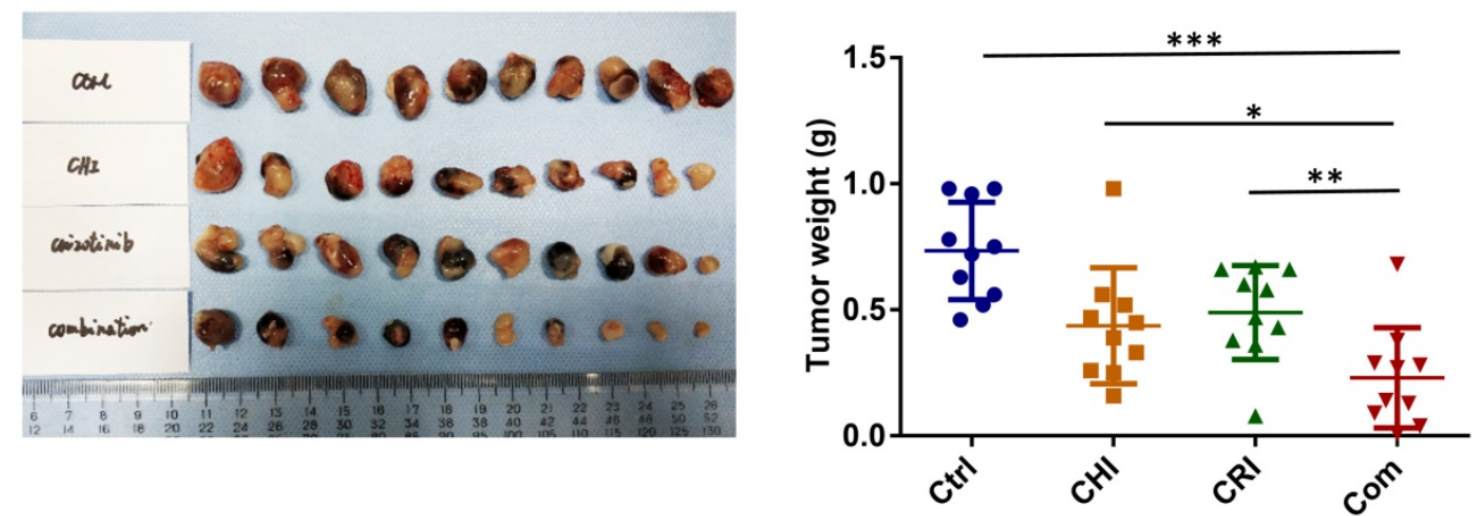

B
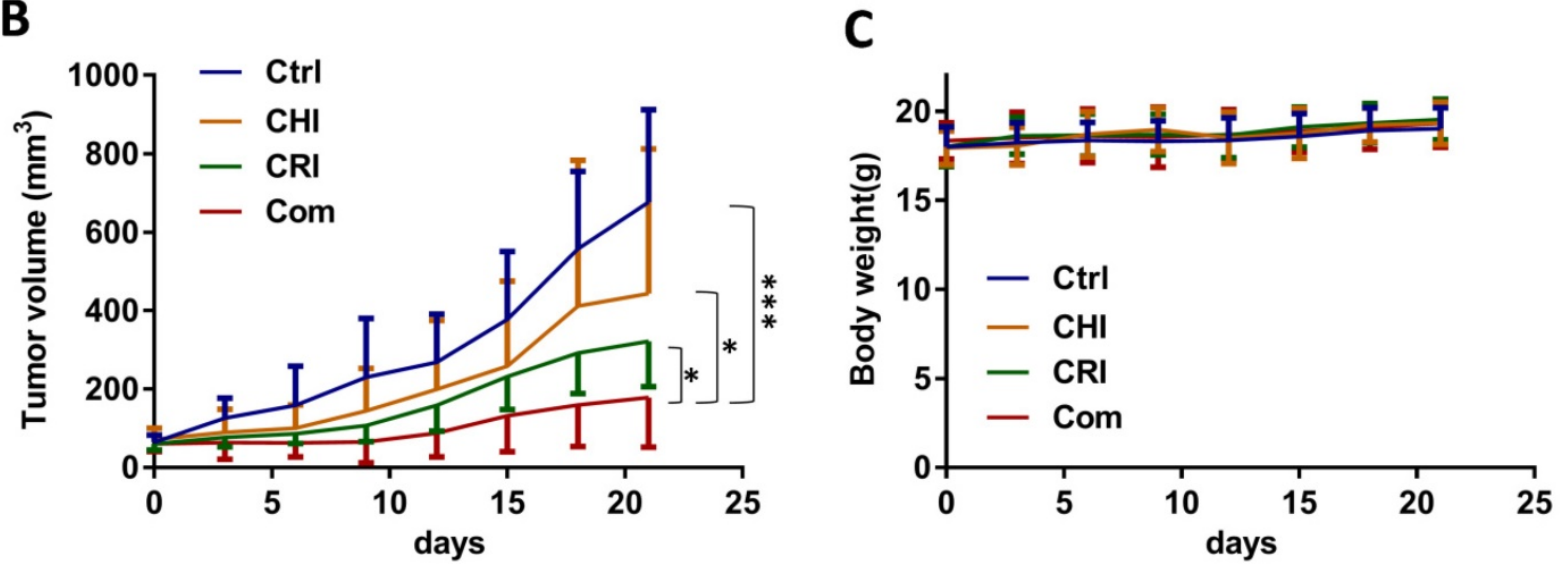

Figure 8. Effect of the combined treatment of chidamide and crizotinib on the growth of HCC827-derived tumor xenografts in Balb/c nude mice. (A) The growth status of $\mathrm{HCC} 827$ xenografts in mice treated with chidamide, crizotinib, or their combination $(n=10)$ on the 21 st day of treatment. Balb/c nude mice bearing established HCC827-derived tumors were treated with vehicle, chidamide $(5 \mathrm{mg} / \mathrm{kg} / \mathrm{d})$, crizotinib $(25 \mathrm{mg} / \mathrm{kg} / \mathrm{d})$ or drug combinations by intragastric administration daily for 21 days. (B) Tumor volumes in different groups. (C) Body weights of mice in different groups. */*****, $\mathrm{P}<0.05 / 0.01 / 0.001$ 
for why c-MET overexpression is not a biomarker for treatment with crizotinib $[5,43,44]$. Interestingly, we found that NSCLC cells overexpressing c-MET were sensitive to chidamide-crizotinib cotreatment in vitro and in vivo. According to CCLE transcriptome data, NSCLC cell lines used in the present study do not express endogenous HGF [22]. We found that the synergetic chidamide-crizotinib effect was dependent on HGF. Thus, it is useful to study whether the level of serum HGF might associate with the synergetic chidamide-crizotinib effect in patients and chidamide could increase the sensitivity of other kinds of cancer cells to crizotinib treatment.

Previous studies have demonstrated that the inhibition of HDAC could reduce the expression of c-MET and the phosphorylation of extracellular signal-regulated kinases 1 and 2 by decreasing the levels of $h s a-m i R-449 a$ in hepatocellular carcinoma cells [43]. HDACIs, including chidamide and YF454A, could downregulate the expression of c-MET and sensitize the effect of EGFR TKIs on the proliferation of NSCLC cells [17, 32]. HCC827 cells contain mutant EGFR gene, which may lead to primary resistance to crizotinib due to remained activity of mutant EGFR in these cells. It was reported that erotinib, an EGFR inhibitor, could increase the sensitivity of cell to crizotinib [46]. To study whether chidamide might increase the crizotinib sensitivity through downregulation of EGFR phosphorylation, we downregulated EGFR expression in HCC827 cells by siRNA. We found that the synergetic effect of chidamide-crizotinib could not be abolished by the downregulation of EGFR expression (Figure S8A and S8B), although chidamide, crizotinib, and their combination indeed partially decrease the phosphorylation level of EGFR protein in HCC827 cells (Figure S8C) and its derived tumors (Figure S8D). These results suggest that the synergetic effect may not depend on the decrease of EGFR phosphorylation. Instead, the synergetic effect of chidamide-crizotinib should depend on the downregulation of $c-M E T$ expression.

In the present study, we found that chidamide treatment could decrease the amount of c-MET protein, but not c-MET mRNA, in several NSCLC cell lines. Treatment with the proteasome inhibitor MG132 did not block the decrease in c-MET protein in these cells. However, the ribosome inhibitor CHX treatment completely blocked the decrease in c-MET protein by chidamide. These phenomena suggest that chidamide may posttranscriptionally downregulate c-MET expression.

RNA m6A modification discovered in the 1970s is a main modification of eukaryotic messenger RNAs (mRNAs), microRNAs (miRNAs), and long noncoding RNAs (lncRNAs) that regulates gene expression via changes in RNA splicing, translation efficiency, and stability [28, 44-46]. Our results showed that chidamide could downregulate the m6A level of total RNA. The results of the MeRIP-based qRT-PCR analyses further demonstrated that the c-MET m6A copy number was also significantly decreased by chidamide in chidamide-crizotinibsensitive cell lines but not in chidamidecrizotinib-resistant cell lines. Expression changes of RNA m6A methylation-related enzymes are closely related to resistance of cancer cells to chemotherapy and radiotherapy [47]. Further study showed that chidamide could downregulate the levels of m6A methyltransferase METTL3 and WTAP proteins. That the m6A levels of METTL3 and WTAP mRNAs were also decreased by chidamide implies that there might be a positive feedback loop in these cells that controls the homeostasis of m6A modification for adaptive responses of cells to environmental factors, including chidamide. siRNA knockdown of METTL3 and WTAP expression completely abolished the synergistic effect between chidamide and crizotinib. These results indicate that treatment with chidamide can repress methyltransferase METTL3 and WTAP expression and decrease the c-MET protein level through the loss of c-MET mRNA m6A methylation. The underlying mechanism of the downregulation of METTL3 and WTAP expression by chidamide is worth further studying.

The inhibition of HDAC6 by ricolinostat could also downregulate c-MET expression while promoting c-MET ubiquitination-dependent degradation in human diffuse large B-cell lymphoma [20]. In the present study, we also observed that ricolinostat could increase the crizotinib sensitivity of NSCLC cells (HCC827 and H661) with the high level of c-MET expression, but not NSCLC cells (H1650 and A549) with the low level of c-MET expression (Figure S9). However, no effect of ricolinostat on global and c-MET mRNA methylation nor on the expression levels of RNA m6A modifiers were found in NSCLC cells (Figure S10). The above phenomena suggest that HDAC inhibitors might affect the sensitivity of crizotinib in a cancer-type-dependent mechanism. Whether other HDAC inhibitors could be used to increase the sensitivity of NSCLC cells to crizotinib needs to study further.

In conclusion, the present study demonstrates that chidamide can decrease the level of c-MET expression by inhibiting mRNA m6A methylation and can subsequently increase the sensitivity, or even overcome the primary resistance of c-MET-expressing NSCLC cells to crizotinib. The combination of chidamide and crizotinib may be a promising novel 
strategy for the treatment of NSCLC with high levels of c-MET expression or c-MET gene amplification. Clinical trials should be conducted to prove our findings.

\section{Abbreviations}

NSCLC: non-small cell lung cancer; HGF: the hepatocyte growth factor; HDACI: histone deacetylase inhibitor; MeRIP: m6A methylated RNA immunoprecipitation assay; IC50: the half maximal inhibitory concentration; CI: the combination index; CCLE: the Cancer Cell Line Encyclopedia; CHX: cycloheximide; ActD: actinomycin D.

\section{Supplementary Material}

Supplementary figures and tables.

http://www.ijbs.com/v16p2595s1.pdf

\section{Acknowledgements}

This work was financially supported by a grant from the Beijing Municipal Commission of Health and Family Planning (PXM2018_026279_000005) to DD. We thank Dr. Xiangping Lu Shenzhen Chipscreen Biosciences Ltd. (Shenzhen, China) for kindly providing us chidamide.

\section{Competing Interests}

The authors have declared that no competing interest exists.

\section{References}

1. Siegel RL, Miller KD, Jemal A: Cancer statistics, 2019. CA: a cancer journal for clinicians 2019, 69(1):7-34.

2. Christensen JG, Zou HY, Arango ME, Li Q, Lee JH, McDonnell SR, Yamazaki S, Alton GR, Mroczkowski B, Los G: Cytoreductive antitumor activity of PF-2341066, a novel inhibitor of anaplastic lymphoma kinase and c-Met, in experimental models of anaplastic large-cell lymphoma. Molecular cancer therapeutics 2007, 6(12 Pt 1):3314-3322.

3. Zou HY, Li Q, Lee JH, Arango ME, McDonnell SR, Yamazaki S, Koudriakova TB, Alton G, Cui JJ, Kung PP et al: An orally available small-molecule inhibitor of c-Met, PF-2341066, exhibits cytoreductive antitumor efficacy through antiproliferative and antiangiogenic mechanisms. Cancer research 2007, 67(9):4408-4417.

4. Bergethon K, Shaw AT, Ou SH, Katayama R, Lovly CM, McDonald NT, Massion PP, Siwak-Tapp C, Gonzalez A, Fang R et al: ROS1 rearrangements define a unique molecular class of lung cancers. Journal of clinical oncology 2012, 30(8):863-870.

5. Paik PK, Drilon A, Fan PD, Yu H, Rekhtman N, Ginsberg MS, Borsu L, Schultz $\mathrm{N}$, Berger MF, Rudin CM et al: Response to MET inhibitors in patients with stage IV lung adenocarcinomas harboring MET mutations causing exon 14 skipping. Cancer discovery 2015, 5(8):842-849.

6. Takahashi T, Sonobe M, Kobayashi M, Yoshizawa A, Menju T, Nakayama E, Mino N, Iwakiri S, Sato K, Miyahara R et al: Clinicopathologic features of non-small-cell lung cancer with EML4-ALK fusion gene. Annals of surgical oncology 2010, 17(3):889-897.

7. Dagogo-Jack I, Shaw AT: Crizotinib resistance: implications for therapeutic strategies. Annals of oncology 2016, 27 Suppl 3:iii42-iii50.

8. Falkenberg KJ, Johnstone RW: Histone deacetylases and their inhibitors in cancer, neurological diseases and immune disorders. Nature reviews Drug discovery 2014, 13(9):673-691.

9. Bolden JE, Peart MJ, Johnstone RW: Anticancer activities of histone deacetylase inhibitors. Nature reviews Drug discovery 2006, 5(9):769-784.

10. Shi Y, Dong M, Hong X, Zhang W, Feng J, Zhu J, Yu L, Ke X, Huang H, Shen Z et al: Results from a multicenter, open-label, pivotal phase II study of chidamide in relapsed or refractory peripheral T-cell lymphoma. Annals of oncology 2015, 26(8):1766-1771.
11. Libby EN, Becker PS, Burwick N, Green DJ, Holmberg L, Bensinger WI: Panobinostat: a review of trial results and future prospects in multiple myeloma. Expert review of hematology 2015, 8(1):9-18.

12. Thompson CA: Vorinostat approved for rare lymphoma. American journal of health-system pharmacy 2006, 63(22):2168.

13. Qiu T, Zhou L, Zhu W, Wang T, Wang J, Shu Y, Liu P: Effects of treatment with histone deacetylase inhibitors in solid tumors: a review based on 30 clinical trials. Future oncology (London, England) 2013, 9(2):255-269.

14. Tang SW, Thomas A, Murai J, Trepel JB, Bates SE, Rajapakse VN, Pommier Y: Overcoming Resistance to DNA-Targeted Agents by Epigenetic Activation of Schlafen 11 (SLFN11) Expression with Class I Histone Deacetylase Inhibitors. Clinical cancer research 2018, 24(8):1944-1953.

15. Chen SY, Zheng XW, Cai JX, Zhang WP, You HS, Xing JF, Dong YL: Histone deacetylase inhibitor reverses multidrug resistance by attenuating the nucleophosmin level through PI3K/Akt pathway in breast cancer. International journal of oncology 2016, 49(1):294-304.

16. Nimmanapalli R, Fuino L, Bali P, Gasparetto M, Glozak M, Tao J, Moscinski L, Smith C, Wu J, Jove R et al: Histone deacetylase inhibitor LAO824 both lowers expression and promotes proteasomal degradation of Bcr-Abl and induces apoptosis of imatinib mesylate-sensitive or -refractory chronic myelogenous leukemia-blast crisis cells. Cancer research 2003, 63(16):5126-5135.

17. Yu W, Lu W, Chen G, Cheng F, Su H, Chen Y, Liu M, Pang X: Inhibition of histone deacetylases sensitizes EGF receptor-TK inhibitor-resistant non-small-cell lung cancer cells to erlotinib in vitro and in vivo. British Journal of Pharmacology 2017, 174(20):3608-3622.

18. Chen MC, Chen $\mathrm{CH}$, Wang JC, Tsai AC, Liou JP, Pan SL, Teng CM: The HDAC inhibitor, MPT0E028, enhances erlotinib-induced cell death in EGFR-TKI-resistant NSCLC cells. Cell Death \& Disease 2013, 4:e810.

19. Greve G, Schiffmann I, Pfeifer D, Pantic M, Schuler J, Lubbert M: The pan-HDAC inhibitor panobinostat acts as a sensitizer for erlotinib activity in EGFR-mutated and -wildtype non-small cell lung cancer cells. BMC cancer 2015, 15:947.

20. Liu Z, Cai Y, Yang Y, Li A, Bi R, Wang L, Shen X, Wang W, Jia Y, Yu B et al: Activation of MET signaling by HDAC6 offers a rationale for a novel ricolinostat and crizotinib combinatorial therapeutic strategy in diffuse large B-cell lymphoma. The Journal of pathology 2018, 246(2):141-153.

21. Shen J, Najafi S, Stable S, Fabian J, Koeneke E, Kolbinger FR, Wrobel JK, Meder B, Distel M, Heimburg T et al: A kinome-wide RNAi screen identifies ALK as a target to sensitize neuroblastoma cells for HDAC8-inhibitor treatment. Cell death and differentiation 2018, 25(12):2053-2070.

22. Ning ZQ, Li ZB, Newman MJ, Shan S, Wang XH, Pan DS, Zhang J, Dong M, Du X, Lu XP: Chidamide (CS055/HBI-8000): a new histone deacetylase inhibitor of the benzamide class with antitumor activity and the ability to enhance immune cell-mediated tumor cell cytotoxicity. Cancer chemotherapy and pharmacology 2012, 69(4):901-909.

23. Chou TC: Theoretical basis, experimental design, and computerized simulation of synergism and antagonism in drug combination studies. Pharmacological Reviews 2006, 58(3):621-681.

24. Baschieri F, Dayot S, Elkhatib N, Ly N, Capmany A, Schauer K, Betz T, Vignjevic DM, Poincloux R, Montagnac G: Frustrated endocytosis controls contractility-independent mechanotransduction at clathrin-coated structures. Nature communications 2018, 9(1):3825.

25. Ping XL, Sun BF, Wang L, Xiao W, Yang X, Wang WJ, Adhikari S, Shi Y, Lv Y, Chen YS et al: Mammalian WTAP is a regulatory subunit of the RNA N6-methyladenosine methyltransferase. Cell research 2014, 24(2):177-189.

26. Huang Z, Zhou W, Li Y, Cao M, Wang T, Ma Y, Guo Q, Wang X, Zhang C, Zhang $C$ et al: Novel hybrid molecule overcomes the limited response of solid tumours to HDAC inhibitors via suppressing JAK1-STAT3-BCL2 signalling. Theranostics 2018, 8(18):4995-5011.

27. Marum L: Cancer Cell Line Encyclopedia launched by Novartis and Broad Institute. Future Medicinal Chemistry 2012, 4(8):947.

28. Chen XY, Zhang J, Zhu JS: The role of $\mathrm{m}(6) \mathrm{A}$ RNA methylation in human cancer. Molecular cancer 2019, 18(1):103.

29. Trovato M, Torre ML, Ragonese M, Simone A, Scarfi R, Barresi V, Giuffre G, Benvenga S, Angileri FF, Tuccari G et al: HGF/c-met system targeting PI3K/AKT and STAT3/phosphorylated-STAT3 pathways in pituitary adenomas: an immunohistochemical characterization in view of targeted therapies. Endocrine 2013, 44(3):735-743.

30. Peruzzi B, Bottaro DP: Targeting the c-Met signaling pathway in cancer. Clinical cancer research 2006, 12(12):3657-3660

31. Gong K, Xie J, Yi H, Li W: CS055 (Chidamide/HBI-8000), a novel histone deacetylase inhibitor, induces G1 arrest, ROS-dependent apoptosis and differentiation in human leukaemia cells. The Biochemical journal 2012, 443(3):735-746

32. Zhang N, Liang C, Song W, Tao D, Yao J, Wang S, Ma L, Shi Y, Han X: Antitumor activity of histone deacetylase inhibitor chidamide alone or in combination with epidermal growth factor receptor tyrosine kinase inhibitor icotinib in NSCLC. Journal of Cancer 2019, 10(5):1275-1287.

33. Naldini L, Vigna E, Narsimhan RP, Gaudino G, Zarnegar R, Michalopoulos GK, Comoglio PM: Hepatocyte growth factor (HGF) stimulates the tyrosine kinase activity of the receptor encoded by the proto-oncogene c-MET. Oncogene 1991, 6(4):501-504.

34. Cappuzzo F, Marchetti A, Skokan M, Rossi E, Gajapathy S, Felicioni L, Del Grammastro M, Sciarrotta MG, Buttitta F, Incarbone M et al: Increased MET gene copy number negatively affects survival of surgically resected 
non-small-cell lung cancer patients. Journal of clinical oncology 2009, 27(10):1667-1674.

35. Park S, Choi YL, Sung CO, An J, Seo J, Ahn MJ, Ahn JS, Park K, Shin YK, Erkin OC et al: High MET copy number and MET overexpression: poor outcome in non-small cell lung cancer patients. Histology and histopathology 2012, 27(2):197-207.

36. Tsuta K, Kozu Y, Mimae T, Yoshida A, Kohno T, Sekine I, Tamura T, Asamura H, Furuta K, Tsuda H: c-MET/phospho-MET protein expression and MET gene copy number in non-small cell lung carcinomas. Journal of thoracic oncology 2012, 7(2):331-339.

37. Kong-Beltran M, Seshagiri S, Zha J, Zhu W, Bhawe K, Mendoza N, Holcomb T, Pujara K, Stinson J, Fu L et al: Somatic mutations lead to an oncogenic deletion of met in lung cancer. Cancer research 2006, 66(1):283-289.

38. Ma PC, Tretiakova MS, MacKinnon AC, Ramnath N Johnson C, Dietrich $S$, Seiwert T, Christensen JG, Jagadeeswaran R, Krausz T et al: Expression and mutational analysis of MET in human solid cancers. Genes, chromosomes \& cancer 2008, 47(12):1025-1037.

39. Watermann I, Schmitt B, Stellmacher F, Muller J, Gaber R, Kugler C, Reinmuth N, Huber RM, Thomas M, Zabel P et al: Improved diagnostics targeting c-MET in non-small cell lung cancer: expression, amplification and activation? Diagnostic Pathology 2015, 10:130.

40. Ma PC, Jagadeeswaran R, Jagadeesh S, Tretiakova MS, Nallasura V, Fox EA, Hansen M, Schaefer E, Naoki K, Lader A et al: Functional expression and mutations of c-Met and its therapeutic inhibition with SU11274 and small interfering RNA in non-small cell lung cancer. Cancer research 2005, 65(4):1479-1488.

41. Tsao MS, Liu N, Chen JR, Pappas J, Ho J, To C, Viallet J, Park M, Zhu H: Differential expression of Met/hepatocyte growth factor receptor in subtypes of non-small cell lung cancers. Lung cancer 1998, 20(1):1-16.

42. Ichimura E, Maeshima A, Nakajima T, Nakamura T: Expression of c-met/HGF receptor in human non-small cell lung carcinomas in vitro and in vivo and its prognostic significance. Japanese journal of cancer research : Gann 1996, 87(10):1063-1069.

43. Buurman R, Gurlevik E, Schaffer V, Eilers M, Sandbothe M, Kreipe H, Wilkens L, Schlegelberger B, Kuhnel F, Skawran B: Histone deacetylases activate hepatocyte growth factor signaling by repressing microRNA-449 in hepatocellular carcinoma cells. Gastroenterology 2012, 143(3):811-820.e815.

44. Desrosiers R, Friderici K, Rottman F: Identification of methylated nucleosides in messenger RNA from Novikoff hepatoma cells. Proc Natl Acad Sci U S A 1974, 71(10):3971-3975.

45. Alarcon CR, Lee $\mathrm{H}$, Goodarzi $\mathrm{H}$, Halberg N, Tavazoie SF: N6-methyladenosine marks primary microRNAs for processing. Nature 2015, 519(7544):482-485.

46. Patil DP, Chen CK, Pickering BF, Chow A, Jackson C, Guttman M, Jaffrey SR: m(6)A RNA methylation promotes XIST-mediated transcriptional repression. Nature 2016, 537(7620):369-373.

47. Xiang MY, Liu WS, Tian W, You AB, Deng DJ: RNA N-6-methyladenosine enzymes and resistance of cancer cells to chemotherapy and radiotherapy. Epigenomics 2020, doi: 10.2217/epi-2019-0358 